\title{
4 Influence of Planting Dates and Nitrogen Fertilization on The Performance of Quinoa Genotypes under Toshka Conditions
}

\author{
A. Awadalla and A. S.M. Morsy \\ Department of Agronomy, Faculty of Agriculture Natural and Resources, Aswan \\ University, Aswan 81528, Egypt..
}

Received: 4/12/2016

Accepted: 19/3/2017

\begin{abstract}
WWO FIELD experiments were conducted at South Valley Farm Research Station, Toshka Region, (ARC), Egypt during 2014/2015 and 2015/2016 seasons to study the effect of planting dates ( $1^{s t}$ Oct. and $1^{\text {st }}$ Nov.) and four N-levels (i.e. $0.0,50,100,150 \mathrm{~kg} \mathrm{~N} / \mathrm{fad}$ ) on growth, yield and its components of three quinoa genotypes, (i.e. Kvlsra-2, Regalona and Q-52). Split-split plot design with three replications was used. The results showed that, the effect of planting dates and also N-levels on all the studied traits was significant in both seasons. Increasing $\mathrm{N}$-levels up to $150 \mathrm{~kg} \mathrm{~N} /$ fad significantly increased all traits, while the maximum nitrogen use efficiency (NUE) values were obtained when quinoa received only $50 \mathrm{~kg} \mathrm{~N} / \mathrm{fad}$ in the two seasons. Regalona genotype recorded the highest values for all studied traits, while the Q-52 genotype gave the highest values for the nitrogen use efficiency in both seasons. On the other hand, the interaction between planting dates and N-levels had significant effect for all studied traits. However, the interaction between planting dates and genotypes was also significant for all characters except No of inflorescences/plant, weight of 1000 seeds and NUE in the $2014 / 2015$ season, No of branches/plant, dry weight /plant, grain and biological yield in the 2015/2016 season. The interaction between N-levels and genotypes had significant effect on all characters, except No of branches/ plant and grain yield as well as the NUE in the both season. The interactions between planting dates, genotypes and N-levels was significant for the plant height and No of branches / plant in both seasons, dry weight/plant in the first season and weight of seeds/plant and biological yield in the second season. Ash content increased with increasing the N-level. Regalona genotype had the highest value of $\mathrm{P}, \mathrm{K}, \mathrm{Ca}$ and $\mathrm{Fe}$ from the obtained results, it could be concluded that planting at $1^{\text {st }}$ Nov., Regalona genotype and treated with $150 \mathrm{~kg} \mathrm{~N} / \mathrm{fad}$ had the highest values of seed yield, protein content for planting quinoa under Toshka condition. Results cleared a strong correlation between the yield and its components. Planting quinoa at $1^{s t}$ Nov. using Regalona genotype which fertilized by $150 \mathrm{~kg} \mathrm{~N} /$ fad resulted the highest values of economic evaluation.
\end{abstract}

Keywords Planting date, Quinoa genotypes, Nitrogen fertilizer levels

\section{INTRODUCTION}

Quinoa (Chenopodium quinoa) a member of the Amaranthaceae family. It's considered a pseudocereal that produces a grain-like seed, which can be sold as a whole grain or used in bread, soups or other uses. In other words, it is basically a "seed" which is prepared and eaten similarly to a grain. It is one of the world's most popular health foods. In Egypt, little information is known about it and it's ideally suited as a potential new crop option for Egyptian producers. Quinoa is gluten-free, high in protein and one of the few plant foods that contain all nine essential amino acids. It is also high in fiber, magnesium, B-vitamins, iron, potassium, calcium, phosphorus, vitamin $\mathrm{E}$ and various beneficial antioxidants. (Repo-Carrasco et al., 2003, Dini et al., 2005, Geerts et al., 2008 and Vega-Galvez et al., 2010).
Quinoa has been selected by FAO as one of the crops destined to offer food security in the next century (FAO 1998, Jacobsen et al., 2003 and Shams, 2010). Increasing yield production is the one way to overcome the increasing demand for the food industry, feeding poultry and livestock as well as many industrial purposes. It is possible, by increasing productivity per unit area through the extensive growing of high yielding genotypes along with the application of improved agronomic package technique. In addition, quinoa could be grown in newly reclaimed desert land. Toshka project is one of the mega projects, which are being executed in south valley of Egypt to cultivate large areas of the desert. FAO (2011) revealed that the quinoa seeds are sown, depending on location, variety, soil moisture and sowing depth. In many countries, quinoa has

\#Corresponding author email: drahmed1122@yahoo.com 
been tested under different climate conditions with varied yield according to sowing dates (Ujiie et al., 2007, Hirich et al., 2014 and Katsunori et al., 2016). Planting date for some quinoa genotypes is one of the main factors which play a prominent role on quinoa production. Variation in germplasm of quinoa is clear in its response to planting date under native conditions of Toshka. Jacobsen (2003) demonstrated that the quinoa is a crop with a range of requirements for humidity and temperature, with different ecotypes adapted to different conditions. Some genotypes of quinoa are grown under conditions of severe drought, suggesting resistance to this adverse factors. (Aguilar \& Jacobsen, 2003 and Aamer et al., 2014) supported these results.

Different genotypes show the different duration of their development stages and also different total growing periods from 126-157 days under European conditions (Jacobsen,1998), 131-200 days in Peru (Flores,1997) and 110-190 days in South America (Jacobsen \& Stolen, 1993).

Nitrogen is a key input element in agriculture that increases yield than other elements (Marschner, 1995). A nitrogen fertilization requirement of quinoa crop is still under study in the world widely because of variability of ecological conditions. Quinoa response to nitrogen addition by not only increase the crop growth and total plant mass production but also the quality of grain (Finch, 1982). Shams (2012) explored the significant effect of different nitrogen rates $(0,90,180,270$ and $360 \mathrm{~kg} \mathrm{~N} / \mathrm{ha}$.) on plant height, grain yield ( $\mathrm{kg} / \mathrm{ha}$.) and biological yield ( $\mathrm{kg} / \mathrm{ha}$.) of quinoa. Basra et al. (2104) reported that, $\mathrm{N}$-levels of $75 \mathrm{~kg} \mathrm{~N} / \mathrm{ha}$ was proved to be the best level for $\mathrm{N}$ supplementation to harvest maximum economic harvest under agro-ecological conditions of Faisalabad. Pospisil et al. (2006), Abou-Amer \& Kamel (2011), Ebrahim et al. (2014) and Hakan (2015) supported these trends.

Chemical analyses reveal the potential of quinoa seed as a valuable ingredient in the preparation of cereal foods having improved nutritional characteristics.

The aim of this investigation was to study the effects of planting date, N-levels and genotypic variation on yield and yield components, nitrogen use efficiency and some biochemical constituents of quinoa grains under Toshka conditions.

\section{MATERIALS AND METHODS}

\section{Location of experiment}

The present investigation was carried out during 2014/2015 and 2015/2016 seasons at Agricultural Experimental Station of Desert Research Center (DRC), located in Toshka Region. It is laying out in the part of the south valley of Egypt, about 1300 and $280 \mathrm{~km}$ south of Cairo and Aswan, respectively, on latitude $22^{\circ} 25^{\prime} \mathrm{N}, 31^{\circ} 05^{\prime} \mathrm{E}$ and elevation $181 \mathrm{~m}$ above the sea level.

\section{Treatment and experimental design}

Because quinoa is the first time to be cultivated in Toshkaregion, South Valley ofEgypt this investigation aimed to identify the suitable planting dates (first of October and first of November), four N-levels $(0,50$, 100 and $150 \mathrm{~kg} \mathrm{~N} / \mathrm{fad}$.) and three quinoa genotypes namely (KVL-SRA2, Regalona and Q-52) and its variation in yield and its components as well as some biochemical constituents under drip irrigation system. The seeds of quinoa genotypes were obtained from Plant Breeding unit, Plant Genetic Resources Department, Desert Research Center, Egypt. The split-split plots design was used, planting dates were assigned in the main plots, N-levels were randomly distributed in sub plots and genotypes were arranged randomly in the sub-subplots, with three replications. The area of each plot was $20 \mathrm{~m}^{2} 4 \mathrm{~m}$ width (4 ridges $100 \mathrm{~cm}$ apart) and $5 \mathrm{~m}$ in length. Seeds of quinoa were sown on one side of drip irrigated ridge in hills spaced $15 \mathrm{~cm}$ apart then thinned to two plants per hill. Plots were kept free of weeds through hand hoeing. The other cultural practices were performed for quinoa production according to the estimated recommendations that were mentioned in the bulletin of the Denimark National Organization (2008).

\section{Land preparation}

The previous crop was maize (Zea mays L.) in both seasons. At soil preparation, $\mathrm{P}, \mathrm{K}$ sources 37.5 $\mathrm{kg} \mathrm{P}_{2} \mathrm{O}_{5}+48 \mathrm{~kg} \mathrm{~K} \mathrm{O} /$ fad (Faddan=4200 $\mathrm{m}^{2}$ ) were applied. Nitrogen fertilizer (supplied from $\mathrm{NH}_{4} \mathrm{NO}_{3}$ $33.5 \%$ ) was applied in four equal doses, the first after four weeks from planting date and the other doses every two weeks, as a solution with irrigation.

\section{Soil analysis}

For the soil characteristics, soil particle distribution, chemical characteristics and fertility conditions of the experimental sites, soil samples were taken from 0-30 and 30-60 cm depth before planting genotypes of quinoa. (Table 1) and were analyzed according to Page (1982) and Soil Survey Staff (1994).

\section{:Weather data}

Climate of Toshka Region during both growth season of quinoa were obtained from meteorological station Toshka CLAC, ARC and shown in Table 2. 
TABLE 1 . Soil particle distribution, chemical characteristics and fertility conditions of the experimental sites at Toshka Region in 2014/15 and 2015/16 seasons.

\begin{tabular}{|c|c|c|c|c|c|c|c|c|c|c|c|}
\hline \multirow{3}{*}{\multicolumn{2}{|c|}{ Soil analysis }} & \multirow{2}{*}{\multicolumn{2}{|c|}{$\frac{2014 / 2015}{\text { Soil depth (cm) }}$}} & \multirow{2}{*}{\multicolumn{2}{|c|}{$\frac{2015 / 2016}{\text { Soil depth (cm) }}$}} & \multirow{3}{*}{\multicolumn{2}{|c|}{ Soil analysis }} & \multirow{2}{*}{\multicolumn{2}{|c|}{$\frac{2014 / 2015}{\text { Soil depth (cm) }}$}} & \multirow{2}{*}{\multicolumn{2}{|c|}{$\frac{2015 / 2016}{\text { Soil depth (cm) }}$}} \\
\hline & & & & & & & & & & & \\
\hline & & $0-30$ & $30-60$ & $0-30$ & $30-60$ & & & $0-30$ & $30-60$ & $0-30$ & $30-60$ \\
\hline \multirow{5}{*}{ 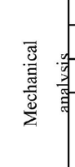 } & Sand $(\%)$ & 67.0 & 51.5 & 65.8 & 51.9 & \multirow{8}{*}{ 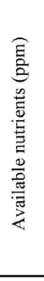 } & $\mathrm{N}$ & 25.00 & 20.00 & 23.00 & 24.00 \\
\hline & Clav (\%) & 3.3 & 9.5 & 3.2 & 9.6 & & $\mathrm{P}$ & 6.00 & 5.00 & 5.50 & 5.50 \\
\hline & Silt (\%) & 29.7 & 39.0 & 31.0 & 38.5 & & $\mathrm{~K}$ & 160.0 & 160.0 & 166.0 & 165.0 \\
\hline & & Sandy & & Sandy loam & & & $\mathrm{Fe}$ & 10.00 & 12.00 & 10.00 & 11.00 \\
\hline & Soil Texture & loam & Loam & & Loam & & $\mathrm{Zn}_{\mathrm{n}}$ & 0.18 & 0.15 & 0.20 & 0.15 \\
\hline \multirow{4}{*}{ 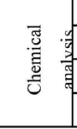 } & $\mathrm{pH}$ & 9.10 & 9.10 & 9.11 & 9.2 & & $\mathrm{Mn}$ & 4.00 & 4.00 & 4.00 & 4.00 \\
\hline & EC $(\%)$ & 0.04 & 0.03 & 0.04 & 0.03 & & $\mathrm{Cu}$ & 0.10 & 0.20 & 0.25 & 0.18 \\
\hline & $\mathrm{CEC}(\mathrm{mg} / 100 \mathrm{~g})$ & 14.80 & 15.00 & 15.00 & 16.00 & & B & 0.80 & 0.90 & 0.80 & 0.80 \\
\hline & $\mathrm{CaCo} 3(\%)$ & 12.80 & 13.80 & 11.90 & 12.10 & & & 0.42 & 0.37 & 0.43 & 0.36 \\
\hline
\end{tabular}

TABLE 2 . Some meteorological parameters for Toshka region during the growing seasons of quinoa 2014/2015 and 2015/2016.*

\begin{tabular}{|c|c|c|c|c|c|c|c|c|c|c|c|c|}
\hline \multirow{3}{*}{ Month } & \multicolumn{6}{|c|}{ 2014/2015 Season } & \multicolumn{6}{|c|}{ 2015/2016 Season } \\
\hline & \multicolumn{2}{|c|}{$\begin{array}{c}\text { Air } \\
\text { temperature }\end{array}$} & \multicolumn{2}{|c|}{$\begin{array}{c}\text { Soil } \\
\text { Temperature }\end{array}$} & \multicolumn{2}{|c|}{$\begin{array}{c}\text { Relative } \\
\text { Humidity }\end{array}$} & \multicolumn{2}{|c|}{$\begin{array}{c}\text { Air } \\
\text { Temperature }\end{array}$} & \multicolumn{2}{|c|}{$\begin{array}{c}\text { Soil } \\
\text { Temperature }\end{array}$} & \multicolumn{2}{|c|}{$\begin{array}{c}\text { Relative } \\
\text { Humidity }\end{array}$} \\
\hline & Min & Max & Min & Max & Min & Max & Min & Max & Min & Max & Min & Max \\
\hline October & 16.4 & 30.1 & 29.7 & 31.1 & 15.9 & 54.9 & 14.7 & 42.7 & 30.8 & 36 & 16.2 & 49.2 \\
\hline November & 8.4 & 33.9 & 25 & 30.6 & 11.8 & 70.1 & 12.5 & 33.3 & 27 & 31.8 & 17.7 & 58.3 \\
\hline December & 8.3 & 31.6 & 22.7 & 27.5 & 15.7 & 84.8 & 6.5 & 27.2 & 22.7 & 28.9 & 24.6 & 67.5 \\
\hline January & 2.4 & 32.9 & 19.8 & 25.9 & 8.9 & 85.1 & 3.2 & 27.8 & 20.8 & 23.8 & 25.2 & 63.9 \\
\hline February & 7.9 & 32.5 & 23 & 26.5 & 9.4 & 87.2 & 3.8 & 33.2 & 21 & 25.6 & 18.1 & 58.6 \\
\hline Marsh & 8.5 & 42.3 & 25.4 & 29.9 & 4.7 & 76.8 & 10 & 43.6 & 24.7 & 29.3 & 12.9 & 54.9 \\
\hline April & 10.4 & 40 & 26.8 & 31.9 & 2.6 & 58.6 & 13.2 & 44.9 & 27.4 & 32.8 & 9.7 & 41.4 \\
\hline May & 16.7 & 45.6 & 30.3 & 34.6 & 1.9 & 74.3 & 17 & 47.1 & 30.9 & 35 & 8.1 & 40.0 \\
\hline June & 19.2 & 45.5 & 32.9 & 35.7 & 4 & 73.2 & 20 & 46.9 & 30.2 & 37.8 & 9.2 & 37.3 \\
\hline
\end{tabular}

*Laboratories unit in Toshka.

\section{Measurements}

Quinoa plants were harvested after 120 days from planting date. Data were recorded on means of ten individual plants with respect to growth characters at the age of 16 weeks which taken at random from each plot representing the three replications. For yield characters at harvest time another sample was assigned for this purpose. The procedure of recording the various data was carried out in the following manner:

Growth, yield and yield attributes characters

Plant height (cm).

Number of branches/plant.

Number of leaves/plant.

Number of inflorescence/plant.

Dry weight g/plant.

Weight of seeds/plant (g).

Weight of 1000 seeds (g).
Seed yield (kg/fad).

Biological yield (kg/fad).

Nitrogen use efficiency (NUE): (kg grain $/ \mathrm{kg}$ $\mathrm{N}$ applied) was calculated according to Craswell \& Godwin (1984) from the following equation:

$\mathrm{NUE}=$ (Grain yield of fertilized plots-Grain yield of unfertilized plots)/(Fertilizer $\mathrm{N}$ applied)

\section{Chemical parameters}

Chemical analysis of grains samples from each replicate of best grain yield treatment were taken in the second season after harvesting and mixed together.

1- Total nitrogen content in grains was determined using the Micro-Kjeldahl method (A.O.A.C., 2000). $\quad$ Protein $\%=$ Total $\mathrm{N} x 6.25$.

2- Determination ofminerals: Iron, Phosphorus and Calcium $(\mathrm{mg} / 100 \mathrm{~g})$ in grain of quinoa were determined according to (Chapman \& Pratt, 1961). Potassium contents (mg/100gm dry matter) were obtained by the method of (Brown \&Lilleland, 1946). 
3- Ash and moisture content $\%$ were determined according to (A.O.A.C, 2000).

\section{Correlation coefficient}

For which parameters and the calculation method.

\section{Economic evaluation}

The economic evaluation included four estimates as follows:

1- Total costs of quinoa production (US \$/ fed): as affected by different treatments

2- $\quad$ Total income $($ US $\$ /$ fad $)=($ Price US $\$$ / ton) $\times$ Yield (ton $/ \mathrm{fad})$.

3- Net farm return (US $\$ /$ fad) $=$ Total Income - Total costs.

4- Benefit/Cost ratio $(\mathrm{B} / \mathrm{C})=$ Total Income/ Total Cost.

All estimation is based on the official and actual market prices determined by FAOStat data, (2014). Economic analysis was done using the method described by CIMMYT (1988).

\section{Statistical analysis}

The collected data were statistically analyzed according to Gomez \& Gomez (1984). The treatment means were compared using LSD test according to Steel \&Torrie (1980).

\section{RESULTS AND DISCUSSION}

\section{Effect of planting dates}

Data in Tables 3 and 4 showed that the effect of planting dates on growth and yield characters as significant in both seasons. The second planting date (i.e. $1^{\text {st }}$ November) had the highest values for all studied traits of yield and its components in the two growing seasons, the corresponding data were plant height (49.01 and $46.00 \mathrm{~cm}$.), No. of branches/plant (28.51 and 28.18), No. of leaves/ plant (95.56 and 88.61), No. of inflorescence / plant (26.83 and 26.85), 1000 seeds weight (4.27 and $3.72 \mathrm{~g}$ ), weight of seeds (35.47 and $33.94 \mathrm{~g}$ ), dry weight (31.66 and $31.83 \mathrm{~g}$ ), grain yield (922.08 and $939.18 \mathrm{~kg} / \mathrm{fad})$, biological yield (1811.55 and $1989.25 \mathrm{~kg} / \mathrm{fad}$ ) and NUE 7.58 and $5.22 \mathrm{~kg} / \mathrm{kg}$ ) in the first and second seasons, respectively. Quinoa plants performed and gave more economical and biological better yield at $1^{\text {st }}$ November than the $1^{\text {st }}$ Oct.

In the light of above result it may be concluded that the first November is good time to quinoa in order to explore its yield potential under Toshka conditions. Similar results were reported by Bertero et al. (2000), Shah \& Akmal (2002), Bertero (2003), Abdel Nour \& Hayam (2011) and Aamer et al. (2014).

\section{Effect of Nitrogen fertilizer levels:}

Data presented in Tables 3 and 4 indicated that the effect of $\mathrm{N}$-levels on yield attributes were significant in the two growing seasons. All studied yield trails except NUE were increased gradually with increasing N-levels from $50 \mathrm{~kg} \mathrm{~N} /$ fad up to $150 \mathrm{~kg} \mathrm{~N} / \mathrm{fad}$, and the differences between them were obvious in growing seasons. Nitrogen at 150 $\mathrm{kg} \mathrm{N} /$ fad produced the maximum values of plant height $(57.75$ and $55.64 \mathrm{~cm}$.), No. of branches/ plant (30.72 and 30.80), No. of leaves/plant (111.45 and 111.70), No. of inflorescence/plant (27.46 and 30.08), 1000 seeds weight (4.75 and $4.63 \mathrm{~g}$ ), weight of seeds ( 36.44 and $36.38 \mathrm{~g}$ ) and dry weight (36.62 and $53.67 \mathrm{~g}$ ) in 2014/2015 and 2015/2016 seasons, respectively.

Data in Table 4 indicated that the effect of $\mathrm{N}$-levels on grain and biological yields was significant in the two seasons. The application of nitrogen fertilizer 50, 100 and $150 \mathrm{~kg} \mathrm{~N} / \mathrm{fad}$ increased grain and biological yields compared with control treatment by $(52.3,58.6$ and $61.9 \%)$ and $(52.9,59.7$ and $63.4 \%$ ) for grain and biological yields in $1^{\text {st }}$ season and by $(36.8,45.8$ and $52.5 \%$ ) and (48.1, 54.4 and 58.7\%) for grain and biological yields in $2^{\text {nd }}$ season. The increase in growth and yield attributes characters gradually with increasing N-levels may be attributed to the role of nitrogen in improving quinoa growth by enhancement meristematic cell division and expansion (Roggatz et al., 1999 and Basra et al. 2014), activity and metabolic, photosynthesis processes and forming filled grains consequently producing heavier grains (Abou-Amer \& Kamel 2011, Shams, 2012, Basra et al., 2014 and Ebrahim et al., 2014). These results are in agreement with those obtained by Schulte et al. (2005), Kakabouki et al. (2014) and Hakan (2015). Their results demonstrated that quinoa grain yield increased with the increasing of N-levels from 50 to $150 \mathrm{~kg}$ $\mathrm{N} /$ ha. The NUE reached a maximum of 8.69 and $6.15 \mathrm{~kg}$ grains $/ \mathrm{kN}$ applied in the first and second seasons, respectively, when $\mathrm{N}-$ levels was applied at $50 \mathrm{~kg} \mathrm{~N} / \mathrm{fad}$ The application of maximum $\mathrm{N}$ levels may results in poor $\mathrm{N}$ uptake and low NUE due to excessive $\mathrm{N}$ losses and decreased $\mathrm{N}$ utilization efficiency (grain weight produced/unit plant N). These results were supported by Shams 
TABLE 3 . Some yield attributes of three quinoa genotypes as affected by planting dates and different nitrogen levels in 2014/2015 and 2015/2016 seasons.

\begin{tabular}{|c|c|c|c|c|c|c|c|c|c|c|c|}
\hline \multirow{3}{*}{\multicolumn{2}{|c|}{ Treatment }} & \multicolumn{10}{|c|}{ Character } \\
\hline & & \multicolumn{2}{|c|}{$\begin{array}{l}\text { Plant height } \\
\text { (cm) }\end{array}$} & \multicolumn{2}{|c|}{$\begin{array}{c}\text { Number of } \\
\text { branches/plant }\end{array}$} & \multicolumn{2}{|c|}{$\begin{array}{l}\text { Number of } \\
\text { leaves/plant }\end{array}$} & \multicolumn{2}{|c|}{$\begin{array}{c}\text { Number of } \\
\text { inflorescence } \\
\text { /plant }\end{array}$} & \multicolumn{2}{|c|}{$\begin{array}{l}1000 \text { Seeds } \\
\text { weight }(\mathrm{g})\end{array}$} \\
\hline & & $\begin{array}{l}2014 / \\
2015\end{array}$ & $\begin{array}{l}2015 / \\
2016\end{array}$ & $\begin{array}{l}2014 / \\
2015\end{array}$ & $\begin{array}{l}2015 / \\
2016\end{array}$ & $\begin{array}{l}2014 / \\
2015\end{array}$ & $\begin{array}{l}2015 / \\
2016\end{array}$ & $\begin{array}{l}2014 / \\
2015\end{array}$ & $\begin{array}{l}2015 / \\
2016\end{array}$ & $\begin{array}{l}2014 / \\
2015\end{array}$ & $\begin{array}{l}2015 / \\
2016\end{array}$ \\
\hline \multicolumn{12}{|c|}{ Planting dates (A) } \\
\hline \multicolumn{2}{|c|}{1 October } & 39.82 & 38.42 & 22.63 & 22.62 & 79.71 & 74.69 & 16.99 & 19.04 & 3.01 & 2.67 \\
\hline \multicolumn{2}{|c|}{1 November } & 49.01 & 46.00 & 28.51 & 28.18 & 95.56 & 88.61 & 26.83 & 26.85 & 4.27 & 3.72 \\
\hline \multicolumn{2}{|c|}{ L.S.D $(0.05)$} & 0.50 & 1.16 & 0.85 & 0.43 & 1.84 & 0.32 & 0.76 & 0.82 & 0.14 & 0.29 \\
\hline \multicolumn{2}{|c|}{$\mathrm{CV} \%$} & 1.10 & 2.72 & 3.30 & 1.67 & 2.07 & 1.00 & 3.42 & 3.54 & 3.89 & 8.84 \\
\hline \multicolumn{12}{|c|}{$\mathrm{N}$ - levels (Kg/fad) (B) } \\
\hline \multicolumn{2}{|l|}{0.0} & 26.71 & 24.79 & 20.31 & 18.50 & 62.78 & 52.41 & 13.63 & 14.59 & 2.03 & 1.81 \\
\hline \multicolumn{2}{|l|}{50} & 43.09 & 41.08 & 23.96 & 24.34 & 81.71 & 73.96 & 20.73 & 20.46 & 3.46 & 2.91 \\
\hline \multicolumn{2}{|l|}{100} & 50.10 & 47.34 & 27.30 & 27.97 & 94.59 & 88.53 & 25.82 & 26.64 & 4.31 & 3.71 \\
\hline \multicolumn{2}{|l|}{150} & 57.75 & 55.64 & 30.72 & 30.80 & 111.45 & 111.70 & 27.46 & 30.08 & 4.75 & 4.36 \\
\hline \multicolumn{2}{|c|}{ L.S.D $(0.05)$} & 0.66 & 0.95 & 0.61 & 0.86 & 1.52 & 1.07 & 0.46 & 0.35 & 0.16 & 0.13 \\
\hline \multicolumn{2}{|c|}{$\mathrm{CV} \%$} & 2.04 & 3.09 & 3.27 & 4.66 & 2.39 & 1.80 & 2.89 & 2.09 & 6.14 & 5.41 \\
\hline \multicolumn{12}{|c|}{ Genotypes ( C) } \\
\hline \multicolumn{2}{|c|}{ Kvlsra 2} & 43.32 & 42.18 & 26.55 & 27.05 & 92.93 & 88.99 & 21.94 & 22.98 & 3.70 & 3.34 \\
\hline \multicolumn{2}{|c|}{ Regalona } & 50.83 & 48.59 & 26.10 & 25.87 & 89.07 & 83.58 & 23.92 & 25.14 & 4.03 & 3.72 \\
\hline \multicolumn{2}{|c|}{ Q-52 } & 39.08 & 35.87 & 24.07 & 23.29 & 80.90 & 72.37 & 19.88 & 20.72 & 3.17 & 2.53 \\
\hline \multicolumn{2}{|c|}{ L.S.D $(0.05)$} & 0.62 & 0.89 & 0.59 & 0.45 & 1.14 & 1.32 & 0.49 & 0.41 & 0.14 & 0.13 \\
\hline \multicolumn{2}{|c|}{$\mathrm{CV} \%$} & 2.37 & 3.55 & 3.89 & 3.00 & 2.19 & 2.73 & 3.76 & 3.05 & 6.73 & 6.99 \\
\hline \multicolumn{12}{|c|}{ Interactions } \\
\hline \multirow{4}{*}{ 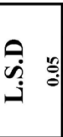 } & $\mathrm{A} \times \mathrm{B}$ & $* *$ & $* *$ & $* *$ & $* *$ & $* *$ & $* *$ & $* *$ & $* *$ & $* *$ & $* *$ \\
\hline & $\mathrm{A} \times \mathrm{C}$ & $* *$ & $* *$ & $*$ & NS & $*$ & * & NS & $* *$ & NS & $* *$ \\
\hline & $\mathrm{B} \times \mathrm{C}$ & $* *$ & $* *$ & NS & NS & $* *$ & $* *$ & $*$ & $* *$ & $* *$ & $* *$ \\
\hline & $\mathrm{A} \times \mathrm{B} \times \mathrm{C}$ & $* *$ & $* *$ & NS & NS & $*$ & $* *$ & NS & NS & NS & NS \\
\hline
\end{tabular}

TABLE 4 . Weight of seeds/plant, dry weight/plant, yield of seeds, biological yield and nitrogen use efficiency as affected by planting dates and different nitrogen levels in 2014/2015 and 2015/2016seasons.

\begin{tabular}{|c|c|c|c|c|c|c|c|c|c|c|c|}
\hline \multirow{3}{*}{\multicolumn{2}{|c|}{ Treatment }} & \multicolumn{10}{|c|}{ Character } \\
\hline & & \multicolumn{2}{|c|}{$\begin{array}{l}\text { Weight of } \\
\text { seeds/ } \\
\text { plant (g) }\end{array}$} & \multicolumn{2}{|c|}{$\begin{array}{l}\text { Dry weight/ } \\
\text { plant (g) }\end{array}$} & \multicolumn{2}{|c|}{$\begin{array}{l}\text { Yield of seeds } \\
\quad(\mathrm{kg} / \mathrm{fad})\end{array}$} & \multicolumn{2}{|c|}{$\begin{array}{c}\text { Biological } \\
\text { Yield (kg/fad) }\end{array}$} & \multicolumn{2}{|c|}{$\begin{array}{c}\text { NUE } \\
(\mathrm{kg} / \mathrm{kg})\end{array}$} \\
\hline & & $\begin{array}{l}2014 / \\
2015 \\
\end{array}$ & $\begin{array}{l}2015 / \\
2016 \\
\end{array}$ & $\begin{array}{l}2014 / \\
2015 \\
\end{array}$ & $\begin{array}{l}2015 / \\
2016 \\
\end{array}$ & $\begin{array}{l}2014 / \\
2015 \\
\end{array}$ & $\begin{array}{l}2015 / \\
2016 \\
\end{array}$ & $\begin{array}{l}2014 / \\
2015 \\
\end{array}$ & $\begin{array}{l}2015 / \\
2016 \\
\end{array}$ & $\begin{array}{l}2014 / \\
2015 \\
\end{array}$ & $\begin{array}{l}2015 / \\
2016 \\
\end{array}$ \\
\hline \multicolumn{12}{|c|}{ Planting dates (A) } \\
\hline $1 \mathrm{O}$ & tober & 22.04 & 20.59 & 24.54 & 23.90 & 686.67 & 785.35 & 1621.50 & 1612.53 & 4.79 & 4.28 \\
\hline $1 \mathrm{No}$ & vember & 35.47 & 33.94 & 31.66 & 31.85 & 922.08 & 939.18 & 1811.55 & 1989.25 & 7.58 & 5.22 \\
\hline L.S. & $(0.05)$ & 1.29 & 1.02 & 0.58 & 0.87 & 50.46 & 19.76 & 35.11 & 81.71 & 0.71 & 0.33 \\
\hline $\mathrm{CV}^{0}$ & & 4.43 & 3.69 & 2.05 & 3.07 & 6.19 & 2.26 & 2.02 & 4.48 & 9.83 & 6.08 \\
\hline \multicolumn{12}{|c|}{$\mathrm{N}$ - levels (Kg/fad) (B) } \\
\hline 0.0 & & 19.95 & 18.00 & 18.87 & 18.47 & 395.38 & 528.21 & 823.72 & 955.56 & - & - \\
\hline 50 & & 26.98 & 23.83 & 24.97 & 25.74 & 829.74 & 835.56 & 1749.78 & 1841.11 & 8.69 & 6.15 \\
\hline 100 & & 31.66 & 30.84 & 31.93 & 31.61 & 955.14 & 973.66 & 2042.72 & 2094.83 & 5.60 & 4.45 \\
\hline 150 & & 36.44 & 36.38 & 36.62 & 35.67 & 1037.23 & 1111.63 & 2249.89 & 2312.06 & 4.28 & 3.88 \\
\hline L.S. & $(0.05)$ & 0.78 & 0.80 & 0.58 & 0.40 & 36.32 & 35.56 & 25.17 & 28.14 & 0.24 & 0.51 \\
\hline $\mathrm{CV}^{0}$ & & 3.75 & 4.02 & 2.83 & 2.00 & 6.21 & 5.67 & 2.02 & 2.15 & 5.36 & 15.21 \\
\hline \multicolumn{12}{|c|}{ Genotypes ( C ) } \\
\hline Kvls & $\mathrm{ra} 2$ & 29.10 & 27.54 & 31.22 & 31.69 & 764.53 & 863.71 & 1691.96 & 1775.04 & 5.88 & 4.63 \\
\hline Reg & lona & 31.20 & 30.18 & 28.05 & 27.78 & 849.14 & 919.60 & 1827.92 & 1867.75 & 6.17 & 4.77 \\
\hline Q-5 & & 25.97 & 24.07 & 25.01 & 24.15 & 757.80 & 803.49 & 1629.71 & 1759.88 & 6.52 & 4.84 \\
\hline L.S. & $(0.05)$ & 0.50 & 0.48 & 0.50 & 0.39 & 31.60 & 85.69 & 24.40 & 30.18 & 0.43 & NS \\
\hline $\mathrm{CV}^{0}$ & & 2.95 & 2.98 & 3.02 & 2.38 & 7.71 & 16.79 & 2.40 & 2.84 & 10.09 & 20.63 \\
\hline \multicolumn{12}{|c|}{ Interactions } \\
\hline \multirow{4}{*}{$\begin{array}{c}\stackrel{8}{0} \\
\stackrel{0}{\dot{0}} \\
\dot{0}\end{array}$} & $\mathrm{~A} \times \mathrm{B}$ & $* *$ & $* *$ & $* *$ & $* *$ & $* *$ & $* *$ & $* *$ & $* *$ & $* *$ & $* *$ \\
\hline & $\mathrm{A} \times \mathrm{C}$ & $* *$ & $* *$ & $* *$ & NS & $*$ & NS & $* *$ & NS & $* *$ & NS \\
\hline & $\mathrm{B} \times \mathrm{C}$ & $* *$ & $* *$ & $* *$ & $* *$ & NS & NS & $* *$ & $*$ & NS & NS \\
\hline & $A \times B \times C$ & NS & $* *$ & $*$ & NS & NS & NS & $* *$ & NS & NS & NS \\
\hline
\end{tabular}


(2012) which found that the decrease in NUE with the increasing of N-levels from 90 up to $360 \mathrm{~kg} \mathrm{~N} /$ ha were $30.36,42.62$ and $49.26 \%$ and $20.40,36.43$ and $39.36 \%$ in the $1^{\text {st }}$ and $2^{\text {nd }}$ seasons, respectively. NUE decreases with increasing N-levels (Schulte et al., 2005; Pospisil et al., 2006 and Abou-Amer \& Kamel, 2011).

\section{Genotypes performance}

Genotypes caused significant effects on quinoa yield attributes in both seasons as shown from results presented in Tables 3 and 4. Kvlsra-2 genotype significantly surpassed other studied genotypes in the No. of branches/plant (26.55 and 27.05 and N. of leaves/plant (92.93 and 88.99) in the first and second seasons, respectively. The highest values of plant height (50.83 and 48.59 $\mathrm{cm}$.), No. of inflorescence/plant (23.92 and 25.14/ plant), 100 seed weight (4.03 and $3.72 \mathrm{~g}$ ), weight of seeds (31.20 and $30.18 \mathrm{~g}$ ) and dry weight (31.22 and $31.69 \mathrm{~g}$ ) were obtained from Regalona genotypes in the $1^{\text {st }}$ and $2^{\text {nd }}$ seasons, respectively. The statistical comparison of genotypes indicated that maximum grain yield (849.14 and $919.60 \mathrm{~kg} /$ fad) was recorded by Regalona genotype, followed by Kvlsra-z genotype (764.53 and $863.71 \mathrm{~kg} / \mathrm{fad})$, while minimum grain yield (757.80 and 803.49 $\mathrm{kg} / \mathrm{fad}$ ) was produced by Q-52 genotype in the first and second seasons, respectively (Table 4). The variation among quinoa genotypes in these characters may be due to the genetical variation. These results agreed with Bhargava et al. (2007), and Aamer et al. (2014). Omar et al. (2014) reported that significant differences were detected among all genotypes for all recorded traits.

\section{Effect of Interaction}

Results in Table 5 indicated that different planting dates and nitrogen fertilizer levels had a significant effect on growth, yield and its components of quinoa i.e. in the two seasons. Applying $150 \mathrm{~kg} \mathrm{~N} / \mathrm{fad}$ at the second planting date ( $1^{\text {st }}$ November) gave the highest values of plant height $(61.85$ and $59.00 \mathrm{~cm}$.), No. of branches/plant (34.62 and 34.33), No .of leaves/ plant (122.99 and 122.11), No. of inflorescence/ plant (35.51 and 34.88), 1000 seed weight (5.66 and $5.16 \mathrm{~g}$ ), weight of seeds (44.53 and $45.49 \mathrm{~g}$ ), dry weight (40.94 and $39.80 \mathrm{~g}$ ), grain yield (1217.39 and $1241.54 \mathrm{~kg} / \mathrm{fad}$ ) and biological yield (2320.89 and $2667.01 \mathrm{~kg} / \mathrm{fad}$ ), while the highest value of NUE resulted from application $50 \mathrm{~kg} \mathrm{~N} /$ fad with planting at $1^{\text {st }}$ Nov. (10.51 and $5.79 \mathrm{~kg} \mathrm{~N} / \mathrm{kg}$ ) in the two seasons, respectively. On the other hand, the first planting date $\left(1^{\text {st }}\right.$ October) with untreated nitrogen gave the lowest values for all studied characters in both seasons.

Significant effect of interaction between planting dates and quinoa genotypes was recorded for plant height, No. of branches/plant, No. of leaves/plant, weight of seeds/plant, dry weight, and biological yield in 2014/2015 season and plant height, No. of leaves/ plant, No. of inflorescence/plant, 1000 seed weight of seeds/plant and nitrogen use efficiency in 2015/2016 season (Table 6). Planted Regalona quinoa genotype at $1^{\text {st }}$ November gave superiorities for all the studied characters. Regarding to the interaction effect between planting dates and genotypes on seed yield in the first season, the greatest seed yield of 999.80 $\mathrm{kg}$ /fed was recorded when Regalona genotype was planted on $1^{\text {st }}$ November.

Results showed that the interaction effect between quinoa genotypes and $\mathrm{N}$-levels was significant on plant height, No. of leaves/plant, dry weight/plant, No. of inflorescence/ plant, 1000 seed weight, weight of seeds/plant and biological yield as affected by interaction between different quinoa genotypes and N-levels are presented in Table 7 . The highest values of plant height $(65.3$ and $63.9 \mathrm{~cm})$, No. of leaves/plant (116.0 and 121.0), dry weight (40.53 and $40.98 \mathrm{~g}$ ) No. of inflorescence/plant (29.9 and 32.7) 1000 seed weight (5.21 and $4.92 \mathrm{~g}$ ), weight of seeds (40.0 and $39.5 \mathrm{~g}$ ) and biological yield (2420.50 and $2432.00 \mathrm{~kg} / \mathrm{fad}$ ), were achieved when Regalona genotype was planted and applied $150 \mathrm{~kg} \mathrm{~N} / \mathrm{fad}$, in the 2014-2015 and 2015-2016 seasons, respectively. While, the lowest values of all studied characters were obtained by Q-52 genotype and untreated $\mathrm{N}$-levels in during both seasons.

Data presented in Tables 3 and 4 show significant effects on planting dates $\mathrm{x} N$-levels $\mathrm{x}$ quinoa genotypes on plant height, No. of leaves/ plant in both seasons, dry weight/plant in the first season, weight of seeds/plant and biological yield $(\mathrm{kg} / \mathrm{fad})$ in the second season. Results in Fig. 1 and 2 demonstrated that planted Regalona at $1^{\text {st }}$ November and application of $150 \mathrm{~kg} \mathrm{~N} /$ fad produced the highest plant height (69.69 and $68.07 \mathrm{~cm}$ ) and No. of leaves/plant (129 and 133) in the $1^{\text {st }}$ and $2^{\text {nd }}$ seasons, respectively.

The interaction among three studied factors excreted significant effect on dry weight/plant in 2014/2015 season as graphically illustrated in Fig. 3. The highest dry weight (45.57 g) was obtained from Regalona quinoa planted at the first November and fertilized by $150 \mathrm{~kg} \mathrm{~N} / \mathrm{fad}$ Furthermore, the interaction among planting date of $1^{\text {st }}$ Nov. x Regalona genotype x $150 \mathrm{~kg} \mathrm{~N} / \mathrm{fad}$, 
TABLE 5. Plant height, No. of branches/plant, No. of leaves/plant, No. of inflorescence/plant, seeds 1000 weight, weight of seeds/plant, dry weight/plant, grain yield, biological yield and nitrogen use efficiency as affected by the interaction between planting dates and nitrogen levels in 2014/2015 and 2015/2016 seasons.

\begin{tabular}{|c|c|c|c|c|c|c|c|c|c|c|c|}
\hline \multicolumn{2}{|c|}{ Treatments } & \multicolumn{10}{|c|}{ Characters } \\
\hline \multirow{2}{*}{ 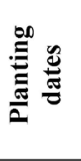 } & \multirow{2}{*}{ 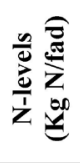 } & \multicolumn{2}{|c|}{$\begin{array}{l}\text { Plant height } \\
\quad \text { (cm) }\end{array}$} & \multicolumn{2}{|c|}{$\begin{array}{c}\text { No. of } \\
\text { branches/plant }\end{array}$} & \multicolumn{2}{|c|}{$\begin{array}{l}\text { No. of leaves/ } \\
\text { Plant }\end{array}$} & \multicolumn{2}{|c|}{$\begin{array}{c}\text { No. of } \\
\text { inflorescence/plant }\end{array}$} & \multicolumn{2}{|c|}{$\begin{array}{c}1000 \text { Seeds } \\
\text { weight }(g)\end{array}$} \\
\hline & & $\begin{array}{l}2014 / \\
2015\end{array}$ & $\begin{array}{l}2015 / \\
2016\end{array}$ & $\begin{array}{l}2014 / \\
2015\end{array}$ & $\begin{array}{l}2015 / \\
2016\end{array}$ & $\begin{array}{l}2014 / \\
2015\end{array}$ & $\begin{array}{l}2015 / \\
2016\end{array}$ & $\begin{array}{l}2014 / \\
2015\end{array}$ & $\begin{array}{c}2015 / 201 \\
6\end{array}$ & $\begin{array}{l}2014 / \\
2015\end{array}$ & $\begin{array}{l}2015 / \\
2016\end{array}$ \\
\hline \multirow{4}{*}{$\begin{array}{c}1 \\
\text { Oct. }\end{array}$} & 0.0 & 20.78 & 22.68 & 18.04 & 15.97 & 55.87 & 49.68 & 11.51 & 12.97 & 1.88 & 1.29 \\
\hline & 50 & 38.52 & 36.53 & 21.18 & 22.88 & 77.43 & 70.18 & 17.17 & 36 & 2.78 & 2.57 \\
\hline & 100 & 46.31 & 42.20 & 23.83 & 24.37 & 85.61 & 77.63 & 19.12 & 2.57 & 3.53 & 3.27 \\
\hline & 150 & 53.65 & 52.28 & 26.81 & 27.30 & 99.91 & 101.29 & 20.17 & 5.29 & 3.85 & 3.57 \\
\hline \multirow{4}{*}{$\begin{array}{c}1 \\
\text { Nov. }\end{array}$} & 0.0 & 32.65 & 26.89 & 21.90 & 21.03 & 69.69 & 55.14 & 15.78 & 16.22 & 2.18 & 2.32 \\
\hline & 50 & 47.67 & 45.64 & 26.74 & 25.80 & 85.98 & 77.73 & 24.29 & 25.57 & 4.14 & 3.26 \\
\hline & 100 & 53.88 & 52.48 & 30.77 & 31.57 & 103.58 & 43 & & 0.72 & 5.09 & 4.14 \\
\hline & 150 & 61.8 & 59.00 & 34.62 & 34.33 & 122.99 & 122.11 & 34.74 & 34.88 & 5.6 & 5.16 \\
\hline \multirow{2}{*}{\multicolumn{2}{|c|}{$\begin{array}{c}\text { L.S.D }(0.05) \\
\text { Treatments } \\
\end{array}$}} & 0.9 & 1.34 & 0.86 & 0.68 & 2.15 & 1.51 & 0.65 & 0.49 & 0.23 & 0.18 \\
\hline & & \multicolumn{10}{|c|}{ Characters } \\
\hline \multirow{2}{*}{ 弱 } & \multirow{2}{*}{ 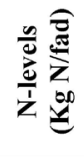 } & \multicolumn{2}{|c|}{$\begin{array}{c}\text { Weight of } \\
\text { seeds/plant (g) }\end{array}$} & \multicolumn{2}{|c|}{$\begin{array}{l}\text { Dry weight/ } \\
\text { Plant (g) }\end{array}$} & \multicolumn{2}{|c|}{$\begin{array}{c}\text { Grain yield } \\
(\mathrm{kg} / \mathrm{fed})\end{array}$} & \multicolumn{2}{|c|}{$\begin{array}{l}\text { Biological } \\
\text { yield }(\mathrm{kg} / \mathrm{fed})\end{array}$} & \multicolumn{2}{|c|}{$\begin{array}{c}\text { NUE } \\
(\mathrm{kg} / \mathrm{kg})\end{array}$} \\
\hline & & $\begin{array}{l}2014 / \\
2015\end{array}$ & $\begin{array}{l}2015 / \\
2016\end{array}$ & $\begin{array}{l}2014 / \\
2015\end{array}$ & $\begin{array}{l}2015 / \\
2016\end{array}$ & $\begin{array}{l}2014 / \\
2015\end{array}$ & $\begin{array}{l}2015 / \\
2016\end{array}$ & $\begin{array}{l}2014 / \\
2015\end{array}$ & $\begin{array}{l}2015 / \\
2016\end{array}$ & $\begin{array}{l}2014 / \\
2015\end{array}$ & $\begin{array}{l}2015 / \\
2016\end{array}$ \\
\hline \multirow{4}{*}{$\begin{array}{c}1 \\
\text { Oct. }\end{array}$} & 0.0 & 15.51 & 14.72 & 16.57 & 16.18 & 372.67 & 499.17 & 801.78 & 880.56 & - & - \\
\hline & 50 & 21.51 & 17.44 & 20.91 & 21.26 & 715.88 & 805.64 & 1590.22 & 1725.78 & 6.86 & 6.13 \\
\hline & 100 & 22.80 & 22.92 & 28.27 & 26.62 & 801.06 & 854.90 & 1915.11 & 1846.67 & 4.28 & 3.56 \\
\hline & 150 & 28.34 & 27.27 & 32.3 & 31.53 & 857.09 & 981.71 & 2178.89 & 1997.11 & 3.23 & 3.15 \\
\hline \multirow{4}{*}{$\begin{array}{c}1 \\
\text { Nov. }\end{array}$} & 0.0 & 24.39 & 21.28 & 21.18 & 20.76 & 418.10 & 557.26 & 845.67 & 1030.56 & - & - \\
\hline & 50 & 32.44 & 30.22 & 29.02 & 30.23 & 943.60 & 865.48 & 1909.33 & 1956.44 & 10.51 & 5.79 \\
\hline & 100 & 40.52 & 38.76 & 35.49 & 36.60 & 1109.22 & 1092.31 & 2170.34 & 2343.10 & 6.91 & 5.31 \\
\hline & 150 & 44.53 & 45.49 & 40.94 & 39.80 & 1217.39 & 1241.54 & 2320.89 & 26267.01 & 5.33 & 4.56 \\
\hline \multicolumn{2}{|c|}{ L.S.D $(0.05)$} & 1.11 & 1.13 & 2.75 & 0.57 & 51.36 & 50.29 & 35.60 & 39.80 & 0.36 & 0.76 \\
\hline
\end{tabular}

TABLE 6. Plant height, No. of branches/plant, No. of leaves/plant, No. of inflorescence/plant, seeds 1000 weight, weight of seeds/plant, dry weight/plant, grain yield, biological yield and nitrogen use efficiency as affected by the interaction between planting dates and genotypes in 2014/2015 and 2015/2016 seasons.

\begin{tabular}{|c|c|c|c|c|c|c|c|c|c|c|c|}
\hline \multicolumn{2}{|c|}{ Treatments } & \multicolumn{10}{|c|}{ Characters } \\
\hline \multirow{2}{*}{$\begin{array}{l}\text { Planting } \\
\text { dates }\end{array}$} & \multirow[t]{2}{*}{ Genotypes } & \multicolumn{2}{|c|}{$\begin{array}{l}\text { Plant height } \\
\quad(\mathrm{cm})\end{array}$} & \multicolumn{2}{|c|}{$\begin{array}{c}\text { No. of } \\
\text { branches/ } \\
\text { Plant } \\
\end{array}$} & \multicolumn{2}{|c|}{$\begin{array}{l}\text { No. of leaves/ } \\
\text { Plant }\end{array}$} & \multicolumn{2}{|c|}{$\begin{array}{c}\text { No. of } \\
\text { inflorescence/plant }\end{array}$} & \multicolumn{2}{|c|}{$\begin{array}{l}1000 \text { Seeds } \\
\text { weight }(g)\end{array}$} \\
\hline & & $\begin{array}{l}2014 / \\
2015\end{array}$ & $\begin{array}{l}2015 / \\
2016\end{array}$ & $\begin{array}{l}2014 / \\
2015\end{array}$ & $\begin{array}{l}2015 / \\
2016\end{array}$ & $\begin{array}{l}2014 / \\
2015\end{array}$ & $\begin{array}{l}2015 / \\
2016\end{array}$ & $\begin{array}{l}2014 / \\
2015\end{array}$ & $\begin{array}{l}2015 / \\
2016\end{array}$ & $\begin{array}{l}2014 / \\
2015\end{array}$ & $\begin{array}{l}2015 / \\
2016\end{array}$ \\
\hline \multirow{3}{*}{$\begin{array}{c}1 \\
\text { Oct. }\end{array}$} & Kvlsra 2 & 38.43 & 38.65 & 23.17 & 24.02 & 84.17 & 82.40 & 18.75 & 20.88 & 32.05 & 2.67 \\
\hline & Regalona & 44.77 & 43.61 & 23.36 & 23.09 & 81.38 & 75.63 & 16.96 & 18.97 & 3.40 & 3.03 \\
\hline & Q-52 & 36.25 & 33.01 & 21.37 & 20.75 & 73.57 & 66.05 & 15.27 & 17.29 & 2.57 & 2.23 \\
\hline \multirow{3}{*}{$\begin{array}{c}1 \\
\text { Nov. }\end{array}$} & Kvlsra 2 & 48.22 & 45.71 & 29.93 & 30.08 & 101.69 & 95.59 & 29.08 & 29.40 & 4.36 & 3.92 \\
\hline & Regalona & 56.90 & 53.56 & 28.83 & 28.65 & 96.76 & 91.53 & 26.93 & 27.00 & 4.67 & 4.41 \\
\hline & Q-52 & 41.92 & 38.73 & 26.77 & 25.83 & 88.23 & 78.69 & 24.48 & 24.14 & 3.77 & 2.83 \\
\hline \multirow{2}{*}{\multicolumn{2}{|c|}{$\begin{array}{l}\text { L.S.D }(0.05) \\
\text { Treatments }\end{array}$}} & 0.88 & 1.26 & 0.83 & NS & 1.61 & 1.86 & NS & 0.59 & NS & 0.19 \\
\hline & & \multicolumn{10}{|c|}{ Characters } \\
\hline \multirow{2}{*}{$\begin{array}{l}\text { Planting } \\
\text { dates }\end{array}$} & \multirow[t]{2}{*}{ Genotypes } & \multicolumn{2}{|c|}{$\begin{array}{l}\text { Weight of } \\
\text { seeds/plant } \\
\text { (g) }\end{array}$} & \multicolumn{2}{|c|}{$\begin{array}{l}\text { Dry weight/ } \\
\text { Plant (g) }\end{array}$} & \multicolumn{2}{|c|}{$\begin{array}{l}\text { Grain yield } \\
\text { (kg/fad) }\end{array}$} & \multicolumn{2}{|c|}{$\begin{array}{c}\text { Biological } \\
\text { yield (kg/fad) }\end{array}$} & \multicolumn{2}{|c|}{$\begin{array}{c}\text { NUE } \\
(\mathrm{kg} / \mathrm{kg})\end{array}$} \\
\hline & & $\begin{array}{l}2014 / \\
2015\end{array}$ & $\begin{array}{l}2015 / \\
2016\end{array}$ & $\begin{array}{l}2014 / \\
2015\end{array}$ & $\begin{array}{l}2015 / \\
2016\end{array}$ & $\begin{array}{l}2014 / \\
2015\end{array}$ & $\begin{array}{l}2015 / \\
2016\end{array}$ & $\begin{array}{l}2014 / \\
2015\end{array}$ & $\begin{array}{l}2015 / \\
2016\end{array}$ & $\begin{array}{l}2014 / \\
2015\end{array}$ & $\begin{array}{l}2015 / \\
2016\end{array}$ \\
\hline \multirow{3}{*}{$\begin{array}{c}1 \\
\text { Oct. }\end{array}$} & Kvlsra 2 & 22.37 & 20.26 & 27.15 & 27.83 & 671.15 & 785.75 & 1605.16 & 1595.50 & 4.84 & 4.15 \\
\hline & Regalona & 24.08 & 23.17 & 24.62 & 23.83 & 704.47 & 745.79 & 1704.58 & 1556.42 & 4.70 & 4.50 \\
\hline & Q-52 & 19.67 & 18.19 & 21.83 & 20.02 & 684.39 & 824.53 & 1554.75 & 1685.67 & 4.83 & 4.18 \\
\hline \multirow{3}{*}{$\begin{array}{c}1 \\
\text { Nov. }\end{array}$} & Kvlsra 2 & 35.83 & 34.82 & 35.30 & 35.54 & 844.44 & 941.67 & 1778.75 & 1954.58 & 6.92 & 5.11 \\
\hline & Regalona & 38.33 & 37.04 & 31.48 & 31.72 & 993.80 & 861.19 & 1951.25 & 1963.34 & 8.34 & 5.04 \\
\hline & Q-52 & 32.27 & 29.95 & 28.19 & 28.27 & 927.99 & 1014.67 & 1704.67 & 2049.83 & 7.50 & 5.51 \\
\hline \multicolumn{2}{|c|}{ L.S.D $(0.05)$} & 0.71 & 0.68 & 0.71 & NS & 51.93 & NS & 34.51 & NS & NS & 0.61 \\
\hline
\end{tabular}


TABLE 7. Plant height, No. of leaves/plant, dry weight/plant, No. of inflorescence/plant, 1000 seeds weight, weight of seeds/plant, and biological yield as affected by the interaction between planting dates and genotypes in 2014/2015 and 2015/2016 seasons..

\begin{tabular}{|c|c|c|c|c|c|c|c|c|c|c|c|c|c|}
\hline \multirow[b]{4}{*}{ Characters } & \multicolumn{12}{|c|}{ Treatments } & \multirow{4}{*}{ 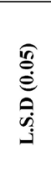 } \\
\hline & \multicolumn{12}{|c|}{ N-levels (kg N/fad) X Genotypes } & \\
\hline & \multicolumn{3}{|c|}{$0.0(\mathrm{~kg} \mathrm{~N} / \mathrm{fed})$} & \multicolumn{3}{|c|}{$50(\mathrm{~kg} \mathrm{~N} / \mathrm{fed})$} & \multicolumn{3}{|c|}{$100(\mathrm{~kg} \mathrm{~N} / \mathrm{fed})$} & \multicolumn{3}{|c|}{$150(\mathrm{~kg} \mathrm{~N} / \mathrm{fad})$} & \\
\hline & 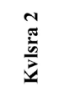 & 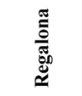 & id & $\frac{N}{\frac{\pi}{5}}$ & 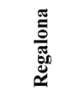 & is & $\begin{array}{l}\frac{\pi}{\pi} \\
\frac{2}{2} \\
\frac{1}{2}\end{array}$ & 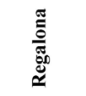 & $\stackrel{\tilde{c}}{\alpha}$ & $\underset{\substack{n \\
\frac{\pi}{2}}}{\frac{N}{2}}$ & 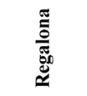 & $\begin{array}{c}\infty \\
0\end{array}$ & \\
\hline \multicolumn{14}{|c|}{$2014 / 2015$ season } \\
\hline Plant height $(\mathrm{cm})$ & 25.2 & 32.8 & 22.1 & 42.1 & 49.7 & 37.4 & 49.0 & 55.5 & 45.7 & 56.9 & 65.3 & 51.1 & 1.25 \\
\hline No. of leaves/plant & 67.1 & 63.6 & 57.5 & 86.9 & 84.9 & 73.4 & 101.7 & 95.7 & 86.4 & 116.0 & 112 & 106.3 & 2.27 \\
\hline Dry weight/plant (g) & 21.05 & 19.08 & 16.48 & 27.42 & 25.22 & 22.27 & 35.90 & 31.53 & 28.35 & 40.53 & 36.38 & 32.95 & 1.01 \\
\hline No. of inflorescence/plant & 15.3 & 13.4 & 12.3 & 22.2 & 21.1 & 18.8 & 28.2 & 25.9 & 23.4 & 29.9 & 27.4 & 25.0 & 0.97 \\
\hline 1000 seeds weight $(\mathrm{g})$ & 1.97 & 2.19 & 1.93 & 3.49 & 3.87 & 3.02 & 4.47 & 4.87 & 3.60 & 4.89 & 5.21 & 4.15 & 0.29 \\
\hline Weight of seeds/plant $(\mathrm{g})$ & 20.1 & 22.1 & 17.7 & 27.3 & 29.2 & 24.5 & 31.8 & 33.6 & 29.6 & 37.2 & 40.0 & 32.1 & 1.00 \\
\hline Biological yield ( $\mathrm{kg} / \mathrm{fad})$ & 783.83 & 872.00 & 815.00 & 1778.50 & 1815.67 & 1655.17 & 1992.50 & 2203.50 & 1932.17 & 2213.00 & 2420.50 & 2116.17 & 49.00 \\
\hline \multicolumn{14}{|c|}{ 2015/2016 season } \\
\hline Plant height $(\mathrm{cm})$ & 24.7 & 28.5 & 21.1 & 42.3 & 47.1 & 33.9 & 47.6 & 54.9 & 39.6 & 54.1 & 63.9 & 48.9 & 1.78 \\
\hline No. of leaves/plant & 57.6 & 53.1 & 46.6 & 79.9 & 76.5 & 65.5 & 97.5 & 89.3 & 78.9 & 121.0 & 115.4 & 98.6 & 2.64 \\
\hline Dry weight/plant (g) & 21.53 & 17.97 & 15.90 & 28.92 & 25.50 & 22.82 & 35.32 & 31.73 & 27.78 & 40.98 & 35.92 & 30.10 & 0.79 \\
\hline No. of inflorescence/plant & 16.1 & 14.7 & 13.0 & 22.5 & 20.4 & 18.5 & 29.3 & 26.7 & 23.9 & 32.7 & 30.2 & 27.4 & 0.83 \\
\hline 1000 seeds weight $(\mathrm{g})$ & 1.79 & 2.18 & 1.45 & 3.07 & 3.52 & 2.15 & 3.90 & 4.27 & 2.95 & 4.60 & 4.92 & 3.57 & 0.26 \\
\hline Weight of seeds/plant $(\mathrm{g})$ & 17.9 & 20.3 & 15.9 & 23.3 & 26.7 & 21.1 & 31.1 & 34.2 & 27.2 & 37.5 & 39.5 & 32.1 & 0.96 \\
\hline Biological yield $(\mathrm{kg} / \mathrm{fed})$ & 954.67 & 1005.00 & 907.00 & 1834.0 & 1894.00 & 1795.23 & 2049.17 & 2140.00 & 2095.34 & 2263.83 & 2432.00 & 2240.23 & 60.35 \\
\hline
\end{tabular}

gave most weight of seeds/plant and biological yield (49.33 $\mathrm{g}$ and $2759.9 \mathrm{~kg} / \mathrm{fad})$, respectively, as shown in Fig. 4 and 5.

Chemical composition of quinoa genotypes in seeds

Results of chemical analysis presented in Table 8 , cleared that the crude protein $\%$ and ash content of quinoa genotypes seeds were increased with increasing N-levels gradually. The highest crude protein $\%$ and ash were associated with application of the highest levels of nitrogen. The highest values of were 13.39 and 14.41 crude protein $\%$ and 3.12 and 3.35 ash content were obtained from Regalona genotype, 150, kg N/ fad and planting date ( $1^{\text {st }}$ Nov.), while the lowest values of crude protein 8.01 and $7.76 \%$ and ash content 2.00 and 2.01 were obtained from Q-52 genotype $\mathrm{x}$ planting date $\left(1^{\text {st }}\right.$ October $)$ with untreated nitrogen in the first and second seasons, respectively. This would apply valuable nutrition value which characterized the quinoa grain rather than other cereal. Also, the same treatment gave the lowest value of moisture $\%$ in quinoa grain was 14.20 and $13.87 \%$, indicated that the obtained results were in harmony with that recorded by Pospisil et al. (2006), Abou-Amer \& Kamel (2011), Shams (2012), Elham (2013) and Ragab et al. (2016). The minerals content (i.e $\mathrm{P}, \mathrm{K}, \mathrm{Ca}$ and $\mathrm{Fe}$ ) in the seeds of treated plants with N-levels $\mathrm{X}$ combination planting dates and genotypes behaved the same as N\% behaved by the treatment.

The highest values were recorded with higher level $\mathrm{N}$ of $150 \mathrm{~kg} \mathrm{~N} / \mathrm{fad}$ in combination with sown Regalona genotype in $1^{\text {st }}$ November. Minerals values were $\mathrm{P}$ (664.07 and 677.32), K (966.02 and 976.87), $\mathrm{Ca}$ (90.88 and 91.78) and $\mathrm{Fe}$ (26.59 and $27.54) \mathrm{mg} / 100 \mathrm{~g}$ in $1^{\text {st }}$ and $2^{\text {nd }}$ seasons, respectively. The lowest values were obtained from untreated nitrogen with sown Q-52 in $1^{\text {st }}$ October in both seasons. It is known that quinoa seed has higher contents of $\mathrm{P}, \mathrm{K}, \mathrm{Ca}$ and $\mathrm{Fe}$ mineral rather than cereal (Johnson \& Ward, 1993) who found that I $\mathrm{kg}$ dry weight of quinoa has more $\mathrm{Ca}(1487 \mathrm{mg})$, Fe (132 mg), K (9267) and Mn (100 mg) than other cereals, such as wheat and barley. These results are in agreement with those obtained by Repo- Carrasco et al. (2003), Abou-Amer \& Kamel (2011), Elham (2013) and Muhammed (2015).

\section{Correlation coefficient among yield and its components}

Grain yield is considered the most accepted criteria for selection. It is a complex trait and joined to the other characters. The correlations among all pairs of characters are presented in Table 9 .

Results indicate that seed yield/fad was negatively and highly significant correlated with plant height ( $\mathrm{r}$ values-0.98 and -0.99 ) weight of seed/plant, 1000 seed weight and biological yield in the first and second seasons. These results could be extended between plant height and each of weight of seeds/plant, $(r=0.96$ and 0.99$) 1000$ seed weight $(r=0.96$ and 0.98$)$ and biological yield ( $\mathrm{r}=0.99$ and -0.92$)$ in 2014-2015 and 20152016 seasons, respectively. Moreover, the highest and positive significant correlation coefficients were obtained between No. of branches/plant and each of No. of leaves/plant, No. of inflorescence/ 

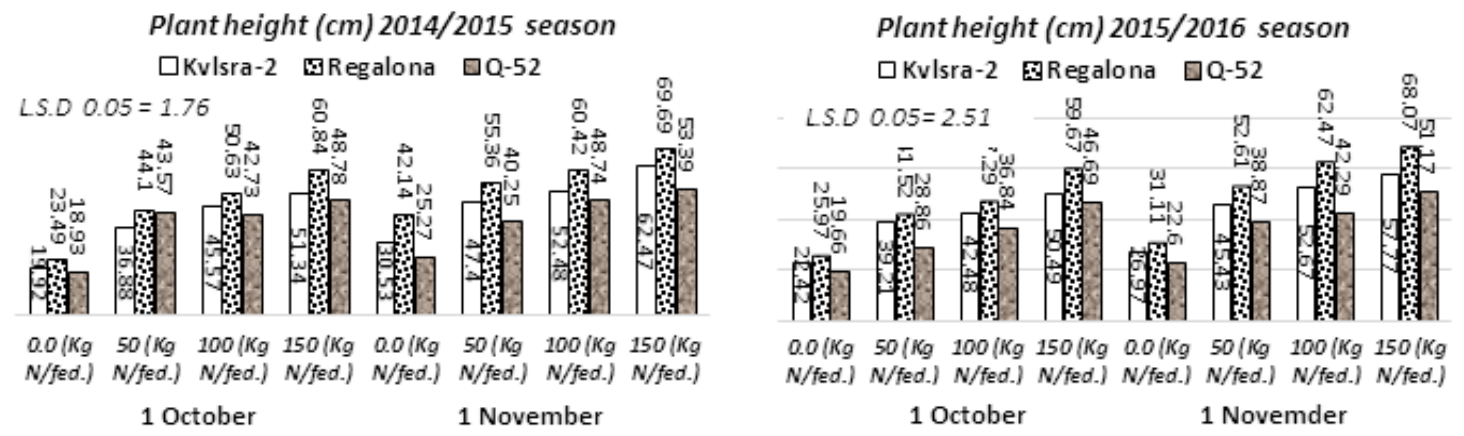

Fig. 1. Plant height $(\mathrm{cm})$ as affected by the interaction among planting dates, $\mathrm{N}$-levels and genotypes during 2014/2015 and 2015/2016 seasons.
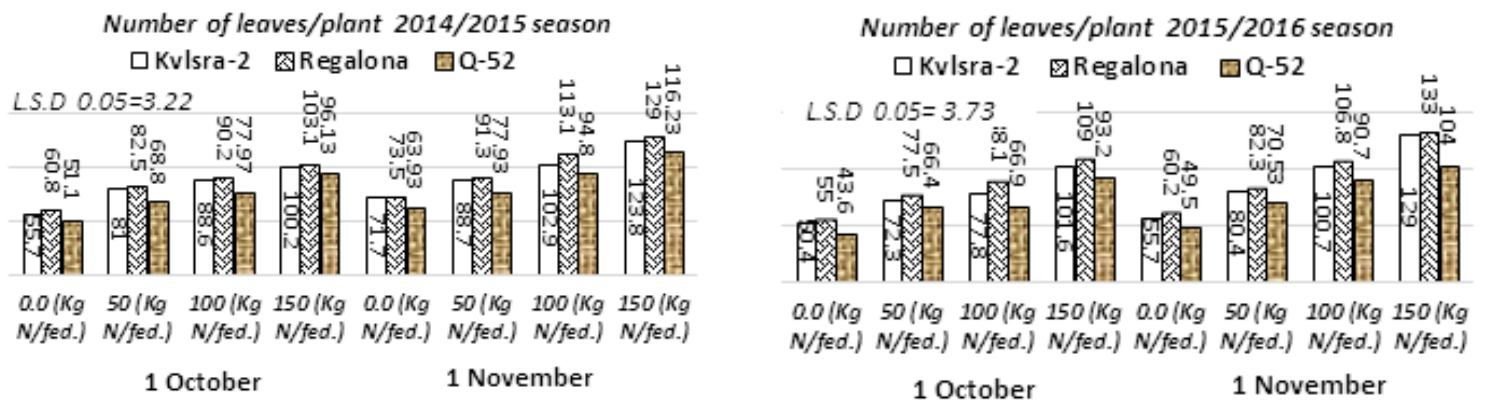

Fig. 2. Number of leaves/plant as affected by the interaction among planting dates, $\mathbf{N}$-levels and genotypes during 2014/2015 and 2015/2016 seasons.

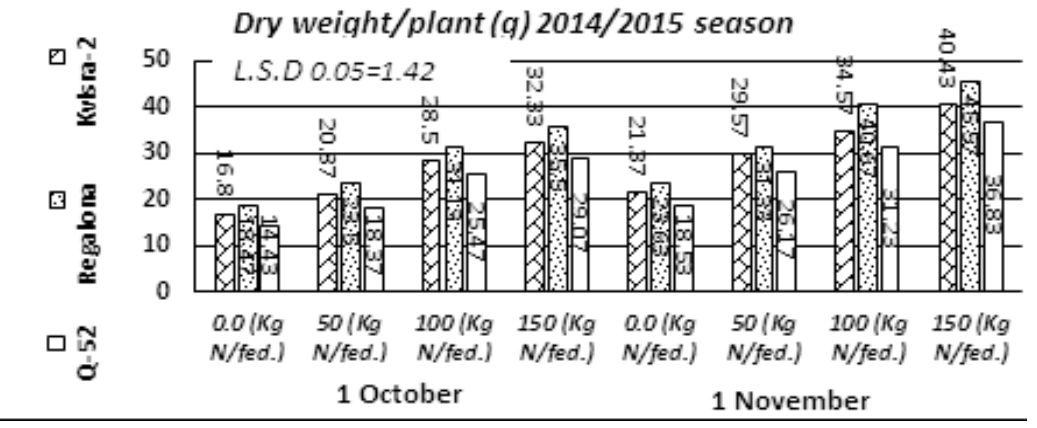

Fig. 3. Dry weight/plant (g) as affected by the interaction among planting dates, $\mathrm{N}$-levels and genotypes during 2014/2015 season.

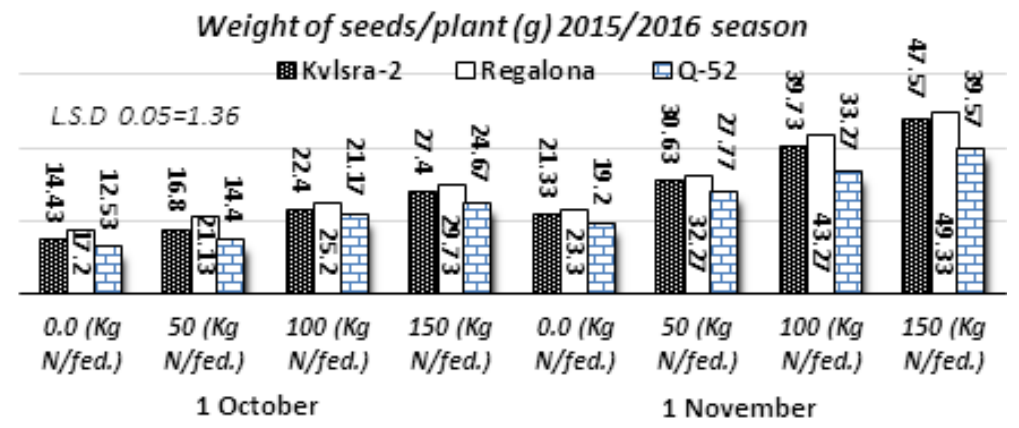

Fig. 4. Weight of seeds/plant (g) as affected by the interaction among planting dates, N-levels and genotypes during 2015/2016 season. 
Biological yield ( $\mathrm{Kg} / \mathrm{fed}$.) 2015/2016 season

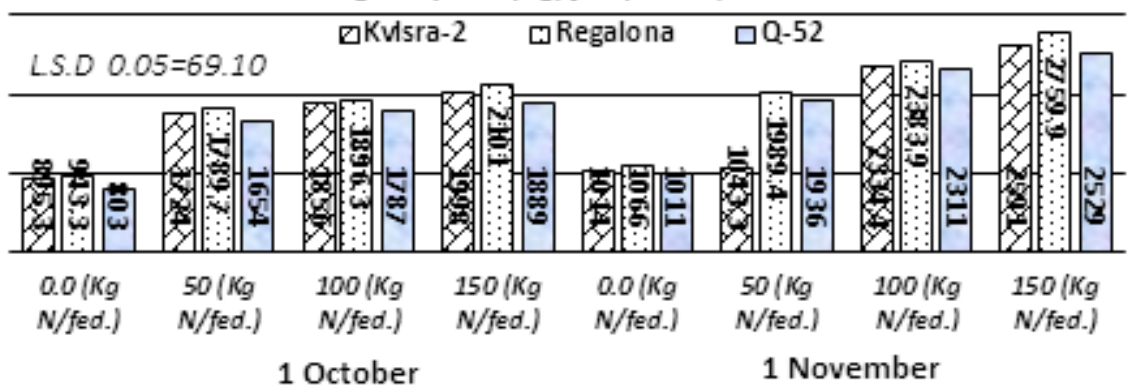

Fig. 5. Biological yield (kg/fad) as affected by the interaction among planting dates, $\mathrm{N}$-levels and genotypes during 2015/2016 season.

TABLE 8. Chemical composition in grains of three quinoa genotypes as affected by planting date and $\mathrm{N}$-levels in 2015/2016 and 2015/2016 seasons.

\begin{tabular}{|c|c|c|c|c|c|c|c|c|c|c|c|c|c|c|c|c|}
\hline \multicolumn{3}{|c|}{ Treatment } & \multicolumn{14}{|c|}{ AS a dry weight basis } \\
\hline \multirow{3}{*}{$\begin{array}{c}\text { Planting } \\
\text { Dates }\end{array}$} & \multirow{3}{*}{ Genotypes } & \multirow{3}{*}{$\begin{array}{l}\text { N-levels } \\
\text { (Kg/fed) }\end{array}$} & \multirow{2}{*}{\multicolumn{2}{|c|}{ Moisture \% }} & \multirow{2}{*}{\multicolumn{2}{|c|}{ Protein \% }} & \multirow{2}{*}{\multicolumn{2}{|c|}{ Ash\% }} & & & & & & $\mathrm{Ca}$ & & e \\
\hline & & & & & & & & & \multicolumn{8}{|c|}{$(\mathrm{mg} / \mathbf{1 0 0 g})$} \\
\hline & & & $\begin{array}{l}2014 / \\
2015\end{array}$ & $\begin{array}{l}2015 / \\
2016\end{array}$ & $\begin{array}{l}2014 / \\
2015\end{array}$ & $\begin{array}{l}2015 / \\
2016\end{array}$ & $\begin{array}{l}2014 / \\
2015\end{array}$ & $\begin{array}{l}2015 / \\
2016\end{array}$ & $\begin{array}{l}2014 / \\
2015\end{array}$ & $\begin{array}{l}2015 / \\
2016\end{array}$ & $\begin{array}{l}2014 / \\
2015\end{array}$ & $\begin{array}{l}2015 / \\
2016\end{array}$ & $\begin{array}{l}2014 / \\
2015\end{array}$ & \begin{tabular}{|l|}
$2015 /$ \\
2016
\end{tabular} & $\begin{array}{l}2014 / \\
2015\end{array}$ & $\begin{array}{l}2015 / \\
2016\end{array}$ \\
\hline \multirow{12}{*}{ 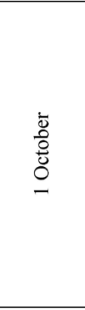 } & \multirow{4}{*}{ Kvlsra2 } & 0.0 & 14.02 & 13.88 & 8.93 & 9.06 & 2.08 & 2.12 & 218.91 & 223.65 & 567.10 & 589.23 & 74.71 & 76.98 & 12.91 & \begin{tabular}{|l|l|}
13.10 \\
\end{tabular} \\
\hline & & 50.0 & 14.10 & 14.06 & 9.63 & 9.75 & 2.17 & 2.26 & 239.93 & 254.01 & 608.13 & 612.20 & 75.01 & 76.99 & 14.00 & 14.06 \\
\hline & & 100.0 & 14.15 & 14.13 & 10.40 & 10.37 & 2.29 & 2.38 & 289.81 & 320.32 & 678.01 & 699.08 & 76.60 & 77.18 & 15.10 & 15.12 \\
\hline & & 150.0 & 14.14 & 14.10 & 10.88 & 11.25 & 2.38 & 2.45 & 301.69 & 398.79 & 744.10 & 776.56 & 77.14 & 77.38 & 16.80 & 16.76 \\
\hline & \multirow{4}{*}{ Regalona } & 0.0 & 14.00 & 13.97 & 7.67 & 8.00 & 2.38 & 2.41 & 221.21 & 232.01 & 599.39 & 620.20 & 76.70 & 77.67 & 13.90 & 14.04 \\
\hline & & 50.0 & 14.11 & 14.06 & 8.83 & 8.45 & 2.44 & 2.49 & 230.05 & 243.21 & 713.41 & 761.00 & 78.30 & 79.09 & 14.10 & 13.11 \\
\hline & & 100.0 & 14.09 & 14.11 & 9.10 & 9.00 & 2.51 & 2.54 & 330.16 & 332.65 & 838.71 & 886.21 & 79.00 & 79.97 & 15.40 & 15.32 \\
\hline & & 150.0 & 14.15 & 14.19 & 9.95 & 10.00 & 2.60 & 2.59 & 411.79 & 432.72 & 899.10 & 912.40 & 79.29 & 80.07 & 16.99 & 17.43 \\
\hline & \multirow{4}{*}{ Q-52 } & 0.0 & 14.93 & 15.76 & 8.01 & 7.76 & 2.00 & 2.01 & 219.17 & 231.01 & 585.35 & 600.87 & 72.78 & 73.37 & 12.99 & 13.04 \\
\hline & & 50.0 & 13.95 & 14.09 & 8.10 & 7.97 & 2.03 & 2.19 & 237.21 & 245.61 & 598.91 & 610.54 & 72.91 & 73.87 & 13.00 & 13.09 \\
\hline & & 100.0 & 14.01 & 13.98 & 9.01 & 8.19 & 2.12 & 2.31 & 278.15 & 297.95 & 683.67 & 689.06 & 73.73 & 74.75 & 14.80 & \begin{tabular}{|l|l|}
14.87 \\
\end{tabular} \\
\hline & & 150.0 & 14.39 & 14.42 & 9.13 & 9.07 & 2.31 & 2.40 & 312.33 & 364.09 & 747.72 & \begin{tabular}{|l|}
753.80 \\
\end{tabular} & 74.41 & 75.09 & 16.03 & 16.12 \\
\hline \multirow{12}{*}{$\begin{array}{l}\bar{\nu} \\
\text { है } \\
\text { Dे } \\
\text { ż }\end{array}$} & \multirow{4}{*}{ Kvlsra2 } & 0 & 14.21 & 14.11 & 10.01 & 9.96 & 2.59 & 2.67 & 341.61 & 376.76 & 831.02 & \begin{tabular}{|l|}
833.43 \\
\end{tabular} & 81.81 & 83.83 & 16.86 & 17.02 \\
\hline & & 50.0 & 14.63 & 14.07 & 11.03 & 10.94 & 2.69 & 2.87 & 419.91 & 447.86 & 906.81 & \begin{tabular}{|l|l|}
945.54 \\
\end{tabular} & 84.99 & 88.78 & 19.90 & 20.43 \\
\hline & & 100.0 & 13.92 & 14.23 & 11.92 & 11.06 & 2.80 & 2.94 & 518.10 & 578.05 & 943.31 & 962.08 & 86.07 & 89.98 & 21.21 & 22.11 \\
\hline & & 150.0 & 14.30 & 14.20 & 12.14 & 12.56 & 2.95 & 3.20 & 629.39 & 643.94 & 958.21 & 969.32 & 88.15 & 90.19 & 23.12 & 24.21 \\
\hline & \multirow{4}{*}{ Regalona } & 0.0 & 14.78 & 15.01 & 11.10 & 10.69 & 2.65 & 2.77 & 410.61 & 446.90 & 876.20 & 889.43 & 85.23 & 86.66 & 18.76 & 19.53 \\
\hline & & 50.0 & 14.17 & 14.14 & 12.14 & 11.88 & 2.86 & 3.01 & 531.55 & 557.98 & 941.15 & \begin{tabular}{|l|l|}
955.67 \\
\end{tabular} & 87.80 & 90.22 & 21.92 & 22.76 \\
\hline & & 100.0 & 13.79 & 14.31 & 12.98 & 13.25 & 2.96 & 3.21 & 597.46 & 623.06 & 959.91 & 967.87 & 89.15 & 91.45 & 23.63 & 24.08 \\
\hline & & 150.0 & 14.06 & 13.87 & 13.39 & 14.41 & 3.1 & 3.35 & 664.07 & 677.32 & 966.02 & 976.87 & 90.88 & 91.78 & 26.59 & 27.34 \\
\hline & \multirow{4}{*}{ Q-52 } & 0.0 & 14.73 & 15.06 & 10.51 & 10.25 & 2.22 & 2.31 & 318.80 & 336.64 & 779.82 & 798.98 & 81.72 & 82.20 & 15.73 & 16.53 \\
\hline & & 50.0 & 14.80 & 14.87 & 11.10 & 10.94 & 2.38 & 2.47 & 410.15 & 425.85 & 845.15 & 887.56 & 82.95 & 84.34 & 18.87 & 19.98 \\
\hline & & 100.0 & 14.00 & 14.08 & 11.61 & 11.50 & 2.59 & 2.68 & 509.91 & 555.95 & 939.91 & 954.21 & 83.32 & 85.13 & 20.52 & 21.66 \\
\hline & & 150.0 & 14.03 & 14.00 & 12.13 & 11.98 & 2.73 & 2.80 & 621.18 & 654.00 & 961.30 & 976.43 & 85.51 & 86.06 & 21.92 & 22.87 \\
\hline
\end{tabular}

plant and weight of seeds/plant in both seasons and accounted values of 0.98 and $0.99,0.94$ and 0.98 and 0.94 and 0.97 in the $1^{\text {st }}$ and $2^{\text {nd }}$ seasons, respectively, while 1000 seed weight was significantly associated with No. of branches with $r$ value being 0.83 and 0.82 in first and second seasons, respectively, and highly significant and positive correlation with biological yield in 2015/2016 season. Number of leaves/plant was positively correlated and highly significant with each of No. of inflorescence ( $\mathrm{r}$ values $=0.98$ and 0.98 ) and dry weight/plant ( $\mathrm{r}$ values $=0.97$ and 0.98 ) in the first and second season, respectively, while it was significant and negative correlation with biological yield.

On the other hand, No. of inflorescence/plant was highly significant and positive correlation with dry weight $(\mathrm{r}$ values $=0.99$ and 0.90$)$ in $1^{\text {st }}$ and $2^{\text {nd }}$ seasons, respectively. Weight of seeds/ plant revealed highly significantly and positively correlated with 1000 seed weight ( $\mathrm{r}$ values $=0.96$ and 0.99 ) in $1^{\text {st }}$ and $2^{\text {nd }}$ seasons, respectively, while it was highly significant and negative correlation with biological yield ( $\mathrm{r}$ values $=-0.94$ and -0.95 ) in 2014/2015 and 2015/2016 seasons, respectively. These results revealed the strong correlation among the yield and its components and in line with those obtained by EL-Degwy (2013) and Omar et al. (2014).

\section{Economic evaluation}

The results in Table 10 showed that the total cost, which calculated as 280.91 US \$/fad fixed cost (land preparation, seeding and planting, irrigation, fertilizers "P+K", weeding, harvesting, transportation and other expenses. Regalona variety gave the highest of total income, net return and $\mathrm{B} / \mathrm{C}$ ration, followed by Kvlsra- 2 and 
TABLE 9. Correlation coefficients between different characteristics of quinoa genotypes season 2014/2015 (above diagonal) and season 2015/2016 (blow diagonal).

\begin{tabular}{|c|c|c|c|c|c|c|c|c|c|}
\hline Characteristics & $(\mathbf{P H})$ & (NB/P) & $(\mathrm{NL} / \mathrm{P})$ & (NI/P) & (DW) & (WS/P) & (SW) & (BY) & (SY) \\
\hline Plant height $(\mathrm{PH})$ & & 0.66 & 0.37 & 0.54 & 0.34 & $0.96^{* *}$ & $0.96^{* *}$ & $0.99^{* *}$ & $-0.98^{* *}$ \\
\hline No. of branches/plant (NB/P) & 0.67 & & $0.98^{* *}$ & $0.94^{* \pi}$ & $0.94^{* *}$ & $0.83^{*}$ & $0.85^{*}$ & 0.62 & 0.75 \\
\hline No. of leaves/plant (NL/P) & 0.66 & $0.99^{* * *}$ & & $0.98^{* * 4}$ & $0.97^{* *}$ & 0.74 & 0.76 & 0.49 & -0.64 \\
\hline No. of inflorescence/plant (NI/P) & 0.501 & $0.98^{k \pi 3}$ & $0.98^{k / 2}$ & & $0.99^{* * 3}$ & 0.60 & 0.62 & 0.45 & -0.48 \\
\hline Dry weight/plant (DW) & 0.48 & $0.97^{* * *}$ & $0.98^{* * *}$ & $0.90^{*}$ & & 0.58 & 0.60 & 0.31 & -0.64 \\
\hline Weight of seeds/plant (WS/P) & $0.99^{\text {** }}$ & 0.73 & 0.72 & 0.58 & 0.55 & & $0.96^{* *}$ & $-0.94^{k *}$ & $-0.98^{* *}$ \\
\hline 1000 seeds weight (SW) & $0.98^{* *}$ & $0.82^{*}$ & 0.80 & 0.68 & 0.65 & $0.99^{* *}$ & & $0.94^{* *}$ & $-0.99^{* *}$ \\
\hline Biological yield $\mathrm{Kg} /$ fed (BY) & $-0.92^{* *}$ & $0.90^{* *}$ & $-0.89^{*}$ & $0.80^{*}$ & 0.78 & $-0.95^{* *}$ & $0.98^{* *}$ & & $-0.98^{* *}$ \\
\hline Seed yield Kg/fed (SY) & $-0.99^{* *}$ & 0.66 & -0.64 & 0.49 & 0.46 & $-0.99^{* *}$ & $-0.97^{* *}$ & $-0.92^{*}$ & \\
\hline
\end{tabular}

TABLE 10. Effect of planting dates, genotypes and $\mathrm{N}$-levels on quinoa yield and economic analysis in (average $1^{\text {st }}$ and $2^{\text {nd }}$ seasons).

\begin{tabular}{|c|c|c|c|c|c|c|c|}
\hline \multicolumn{3}{|c|}{ Treatment } & \multirow{2}{*}{$\begin{array}{c}\text { Grain yield } \\
\text { (ton/fed) }\end{array}$} & \multirow{2}{*}{$\begin{array}{l}\text { Total income } \\
\text { (US \$/fed) }\end{array}$} & \multirow{2}{*}{$\begin{array}{l}\text { Total costs } \\
\text { (US S/fed) }\end{array}$} & \multirow{2}{*}{$\begin{array}{c}\text { Net return } \\
\text { (US \$/fed) }\end{array}$} & \multirow{2}{*}{$\begin{array}{r}\text { Benefit/Cos } \\
\text { ratio }(B / C)\end{array}$} \\
\hline (A) & (B) & (C) & & & & & \\
\hline \multirow{12}{*}{$\begin{array}{l}\overline{0} \\
0 \\
0 \\
\stackrel{0}{0} \\
0 \\
-\end{array}$} & \multirow{4}{*}{$\frac{\overparen{d}}{\underline{D}}$} & 0.0 & 0.322 & 374.76 & 280.91 & 93.85 & 1.33 \\
\hline & & 50.0 & 0.658 & 765.82 & 322.38 & 443.44 & 2.37 \\
\hline & & 100.0 & 0.720 & 837.98 & 363.85 & 474.13 & 2.30 \\
\hline & & 150.0 & 0.802 & 933.42 & 405.32 & 528.10 & 2.30 \\
\hline & \multirow{4}{*}{ 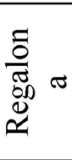 } & 0.0 & 0.355 & 413.17 & 280.91 & 132.26 & 1.47 \\
\hline & & 50.0 & 0.670 & 779.79 & 322.38 & 457.41 & 2.42 \\
\hline & & 100.0 & 0.752 & 875.22 & 363.85 & 511.37 & 2.41 \\
\hline & & 150.0 & 0.846 & 984.63 & 405.32 & 579.31 & 2.43 \\
\hline & \multirow{4}{*}{$\stackrel{v}{d}$} & 0.0 & 0.332 & 374.76 & 286.40 & 88.36 & 1.31 \\
\hline & & 50.0 & 0.656 & 763.49 & 322.38 & 441.11 & 2.37 \\
\hline & & 100.0 & 0.711 & 827.50 & 363.85 & 463.65 & 2.27 \\
\hline & & 150.0 & 0.816 & 949.71 & 405.32 & 544.39 & 2.34 \\
\hline \multicolumn{3}{|c|}{ Mean of A1 } & 0.637 & 740.02 & 344.49 & 395.53 & 2.11 \\
\hline \multirow{12}{*}{ 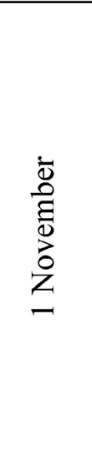 } & \multirow{4}{*}{ 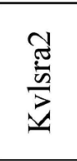 } & 0.0 & 0.370 & 430.63 & 280.91 & 149.72 & 1.53 \\
\hline & & 50.0 & 0.805 & 936.91 & 322.38 & 614.53 & 2.91 \\
\hline & & 100.0 & 1.009 & 1174.33 & 363.85 & 810.48 & 3.22 \\
\hline & & 150.0 & 1.126 & 1310.51 & 405.32 & 905.19 & 3.23 \\
\hline & \multirow{4}{*}{ 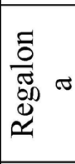 } & 0.0 & 0.414 & 481.84 & 280.91 & 200.93 & 1.72 \\
\hline & & 50.0 & 0.848 & 986.95 & 322.38 & 664.57 & 3.06 \\
\hline & & 100.0 & 1.038 & 1208.09 & 363.85 & 844.24 & 3.32 \\
\hline & & 150.0 & 1.187 & 1381.50 & 405.32 & 976.18 & 3.41 \\
\hline & \multirow{4}{*}{$\stackrel{v}{d}$} & 0.0 & 0.380 & 442.27 & 280.91 & 161.36 & 1.57 \\
\hline & & 50.0 & 0.761 & 885.70 & 322.38 & 563.32 & 2.75 \\
\hline & & 100.0 & 0.956 & 1112.65 & 363.85 & 748.80 & 3.06 \\
\hline & & 150.0 & 1.077 & 1253.48 & 405.32 & 848.16 & 3.09 \\
\hline \multicolumn{3}{|c|}{ Mean of A2 } & 0.831 & 1071.53 & 344.49 & 727.04 & 2.74 \\
\hline \multirow{3}{*}{$\begin{array}{c}\text { Mean } \\
\text { of } B\end{array}$} & \multicolumn{2}{|c|}{ Kvlsra2 } & 0.727 & 846.13 & 343.12 & 503.01 & 2.47 \\
\hline & \multicolumn{2}{|c|}{ Regalona } & 0.764 & 889.19 & 343.12 & 546.07 & 2.59 \\
\hline & \multicolumn{2}{|c|}{$\mathrm{Q}-52$} & 0.711 & 827.50 & 343.12 & 484.38 & 2.41 \\
\hline \multirow{4}{*}{\multicolumn{2}{|c|}{ Mean of C }} & 0.0 & 0.362 & 419.67 & 280.91 & 138.76 & 1.49 \\
\hline & & 50.0 & 0.733 & 853.11 & 322.38 & 530.73 & 2.65 \\
\hline & & 100.0 & 0.864 & 1005.96 & 363.85 & 642.11 & 2.76 \\
\hline & & 150.0 & 0.976 & 1135.54 & 405.32 & 730.22 & 2.80 \\
\hline
\end{tabular}

- Average prevailing market prices of quinoa grains and fertilizers during 2014 to 2016

1 - Price of quinoa grains ( 1 ton $=1163.86$ US $\$$ in Bolivia 2014), source FAOStat, 2014.

2- Fertilizers ( $\left.37.5 \mathrm{~kg} \mathrm{P}_{2} \mathrm{O}_{5} / \mathrm{fad}=25.45 \mathrm{US} \$\right),\left(48 \mathrm{~kg} \mathrm{~K}_{2} \mathrm{O}=73.37 \mathrm{US} \$\right)$ and $(50 \mathrm{~kg} \mathrm{~N}=41.27$ US $\$)$

3- The previous price of fertilizers in Egypt in 2016.

$1($ US $\$)=10.85$ Egyptian pound in $5 / 2016$. 
Q-52. The planting date in $1^{\text {st }}$ November gave the maximum values of economic evaluation. The average of total income for the fad of quinoa yield ranged from about 374.76 US \$ to about 1253.48 US \$ with interaction a $2 \times b 2 \times c 4$ and with interaction $\mathrm{a} 1 \mathrm{xb} 3 \mathrm{xc1}$ as lower and higher values. increases in values of total income, total costs, net return and benefit/cost ratio traits with increasing $\mathrm{N}$-levels up to $150 \mathrm{~kg} \mathrm{~N} /$ fad in average seasons. It might be to apply more nitrogen fertilizer and correlated with increasing in nitrogen levels. These results are in line with those obtained by Jacobsen (2003) and Shams (2012), revealed that the economic analysis results for the farmer depends on the yield and the price to be achieved for the crop and add that any enhanced result will be obtained with either an increased yield or a higher price.

\section{REFERENCES}

Aamer, S., Hassan, M., Ehsanullah, Shakeel, A.A., Mohsin, T. and Aziz, R. (2014) Growth and development of Chenopodium quinoa genotypes at different sowing dates. J. Agric. Res. 52(4), 535-546.

Abdel Nour, Nadya A.R. and Hayam S.A.f. (2011) Influence of sowing date and nitrogen fertilization on yield and its components in some bread wheat genotypes. Egypt. J. Agric. Res. 89(4), 1413-1433.

Abou-Amer, A.I. and Kamel, A.S. (2011) Growth, yield and nitrogen utilization efficiency of quinoa (Chenopodium quinoa) under different rates and methods of nitrogen fertilization. Egypt. J. Agron. 33(2), 155-166.

Aguilar, P.C. and Jacobsen, S.E. (2003) Cultivation of quinoa on the Peruvian Altiplano. Food Rev. Int. 19, 31-41.

A.O.A.C. (2000) Association of Official Agricultural Chemists. "Official Methods of Analysis". (17 $7^{\text {th }}$ ed.), Arlington Virginia 22201, USA.

Basra, S. M. A., Iqbal, S. and Afzal, I. (2014) Evaluating the response of nitrogen application on growth, development and yield of quinoa genotypes. Int.J. Agric. Bio. 16(5), 886-892.

Bhargava, A., Shukla, S. and Ohri, D. (2007) Genetic variability and interrelationship among various morphological and quality traits in quinoa
(Chenopodium quinoa Willd.). Field Crops Research, 101,104-116.

Bertero, H.D. (2003) Response of development processes to temperature and photoperiod in quinoa (Chenopodium quinoa Wild). Food Rev. Int. 19, 87-97.

Bertero, H. D., King, R.W. and Hall, A. J. (2000) Photoperiod and temperature effects on the rate of leaf appearance in quinoa (Chenopodium quinoa). Aust. J. Plant Physiol. 27, 349-356

Brown, J.D. and Lilleland, O. (1946) Rapid determination of Potassium in plant material and extract by flame photometry. Proc. Amer. Soc Hort. Sci. 48,341.345.

Chapman, H.D. and Pratt, R.F. (1961) "Methods for Andysis for Soils". Plant and Water Div Agric. Sci, Univ. of Calif., USA.

CIMMYT (1988) "From Agronomic Data to Farmer Recommendation: an Economic Work Book" D.F: pp.31-33.

Craswell, E.T. and Godwin, D.C. (1984) The efficiency of nitrogen fertilizer applied to cereals in different climates. In " Advances in plant Nutrition "Tinker, P.B. and Luchli, A(Ed) Vol. I. Praeger publ. Country.

Dini, I., Tenore, G.C. and Dini, A. (2005) Nutritional and antinutritional composition of Kancolla seeds: an interesting and underexploited andine food plant. Food Chem. 92,125-132.

Ebrahim, A., Hamid, R.B. and Moral, M. (2014) Effects of ascorbic acid foliar spraying and nitrogen fertilizer management in spraying cultivation of quinoa (Chenopodium quinoa) in North of Iran. Biological forum-An Int. J. 6(2), 254-260

EL-Degwy, I.S. (2013) Performance and genotypic variability of three bread wheat cultivars under stress irrigation regimes. Egypt. J. Agron.35(2), 211-225.

Elham ， F. G. (2013) Effect of nitrogen, phosphorus and biofertilizers on quinoa plant. $J$. Appl. Sci. Res. 9 (8), 5210-5222.

FAOstat (2014) FAOstat data. http://faostat. Fao. org.

FAO, (2011) Quinoa: An ancient crop to contribute to world food security. In: A. Bojanic (Ed.). Regional Office for Latin America and 
the Caribbean, p.63. Food and Agriculture Organization, Rome, Italy.

FAO (1998) Under-Utilized Andean Food Crops. Rome, Italy.

Finch, A. (1982) Fertilizer and fertilization "Introduction and Practical Guide to Crop Fertilization". Weinheim; Deerfield Beach, Florida; Basel: Verlag Chemie.

Flores, F.G. (1997) Estudio Preliminar de la fenologia de la quinoa (Chenopodiumm quinoa willd.). Tesis. Universidad NactionalTecnica del Attopiano, Puno.

Geerts, S., Garcia, M., Cusicanqui, J., Taboada, C., Miranda, R., Yucra, E. and Raes, D.(2008) Review of current knowledge on quinoa (Chenopodium quinoa Wild). Faculty of Agronomy- Agronomic Engineering (Ed.) Universidad Mayor de San Andres, La Paz, Bolivia.

Gomez, K.A. and Gomez, A.A. (1984) "Statistical Procedures for Agricultural Research". $2^{\text {nd }}$ ed., John Wiley Son, New York, USA.

Hakan, G. (2015) Effect of different nitrogen levels on the grain yield and some yield components of quinoa (Chenopodium quinoa Willd) under mediterranean climatic conditions. Turkish Journal of Field Crops, 20(1), 59-64.

Hirich, A., Choukr-Allah, R. and Jacobsen, S.E. (2014) Quinoa in Morocco-Effect of sowing dates on development and yield, J. Agron. Crop Sci. 200, 371-377.

Jacobsen, S.E. (2003) The worldwide potential for quinoa (Chenopodium quinoa Willd.). Food Rev. Int.19(1), 167-177.

Jacobsen, S.E. (1998) Development stability of quinoa under Eurpean conditions. Int. Crops Prod. 7, 169-174.

Jacobsen, S.E. and Stolen, O. (1993) Quinoamorphology and phenology and prospects for its production as a new crop in Europ. Eur. J. Agron. 2,19-29.

Jacobsen, S.E., Mujica,A. and Jensen, C.R. (2003) The resistance of quinoa (Chenopodium quinoa Willd.) to adverse, abiotic factors. Food Rev. Int.19, 99-109.

Johnson, D.L. and Ward, S.M. (1993) In: "Quinoa. New Crops". Janick, J., Simon, J.E.
(Ed.), pp. 222-227 Wiley, New York .

Kakabouki, I., Bilalis, D., Karakanis, A., Zervas, G., Tsiplakou, E. and Hela, D. (2014) Effects of fertilization and tillage system on growth and crude protein content of quinoa (Chenopodium quinoa Wild.): An alternative forage crop, Emir. J. Food Agric. 26(1), 18-24.

Marschner, H. (1995) "Mineral Nutrition of Higher Plants".Academic Press, London, UK.

Katsunori, I., Hikaru, S., Daisuke, O., Yudai, M., Hiroki, H., Misa, M., Syunsuke, K., Masao, H. and Toichi, T. (2016) Effects of sowing time on the seed yield of quinoa (Chenopodium quinoa willd.) in south Kanto, Japan. Agric. Sci. 7, 146153.

Muhammed, A.I. (2015) An assessment of quinoa (Chenopodium quinoa Willd.) potential as a grain crop on marginal lands in Pakistan. AmEuras. J. Agric. \& Environ. Sci. 15(1),16-23.

Omar, S.A., Injy, M.M. and Rasha, M.A. (2014) Genetic evaluation of some quinoa genotypes under Ras Suder conditions. J. Plant Production, Mansoura Univ. 5(11), 1915-1930.

Page, A.L. (1982) "Methods of Soil Analysis. Part 2, Chemical and Microbiological Properties", second ed, Wisconsin, USA.

Pospisil,A., Pospisil, M., Vaga, B. and Svevnjak, S. (2006) Grain yield and protein concentration of two amaranth species as influenced by nitrogen fertilization. Europ. J. Agron. 25, 250-253.

Ragab, S.T., Ayman, H.A.M and Hamdy, A.A. (2016) Effect of biofertilizers as a partial substitute for mineral fertilizer on growth, anatomical structure, mineral elements and yield of wheat under newly reclaimed soil conditions. Int. Curr. Microbial. App. Sci. 5(8),458-469.

Repo-Carrasco, R., Espinoza, C. and Jacobsen, S. (2003) Nutritional value and use of the Andean crops quinoa (Chenopodium quinoa) and Kaniwa (Chenopodium pallidicaule). Food Rev. Int.19,179-189.

Roggatz, U., Mcdonald, A.J.S., Stadenberg, I. and Schrr, U. (1999) Effects of nitrogen deprivation on cell division and expansion in leaves of Ricinus communis Plant Cell Environ.22,81-89.

Schulte, A. E.G., Kaul, H.P., Kruse, M. and Aufhammer, W. (2005) Yield and nitrogen 
utilization efficiency of the pseudo cereals amaranth, quinoa, and buck wheat under differing nitrogen fertilization. Europ. J. Agron. 22(1), 95-100.

Shah, S.M. and Akmal, M. (2002) Effect of different sowing dates on yield and yield components of wheat varieties. Sarhad J. Agric. 18, 143-149.

Shams, A.S. (2010) Combat degradation in rainfed areas by introducing new drought tolerant crops in Egypt. $4^{\text {th }}$ International Conference on Water Resources and Arid Environments, Riyadh, Saudi Arabia, 5-8 December, pp.575-582.

Shams, A.S. (2012) Response of quinoa to nitrogen fertilizer rates under sandy soil conditions. Proc $13^{\text {th }}$ international Conf. Agron., Fac.of Agric., Benha Univ., Egypt, 9-10 September.

Soil Survey Staff (1994) Keys to soil taxonomy.
USDA, Soil conservation Service.

Steel, R.G.D. and Torrie, J.H. (1980) "Principles and Procedures of Statistics, a Biometrical Approach". Mc Graw-Hill Co., $2^{\text {nd }}$ ed., New York, USA.

Ujiie, K, Sasagawa, R., Yamashita, A., Isobe, K., and Ishii, R. (2007) Agronomic studies on quinoa (Chenopodium quinoa willd.) cultivation in Japan-1. Determination of the proper seeding time in the Southern Kanto district for good performance of the grain yield. Japanese J. Crop. Sci. 76, 59-64.

Vega-Gálvez, A., Miranda, M., Vergara, J., Uribe, E., Puente, L. and Martínez, EA. (2010) Nutrition facts and functional potential of quinoa (Chenopodium quinoa willd.), an ancient Andean grain: a review. J.Sci. Food Agric. 90(15), 25412547.

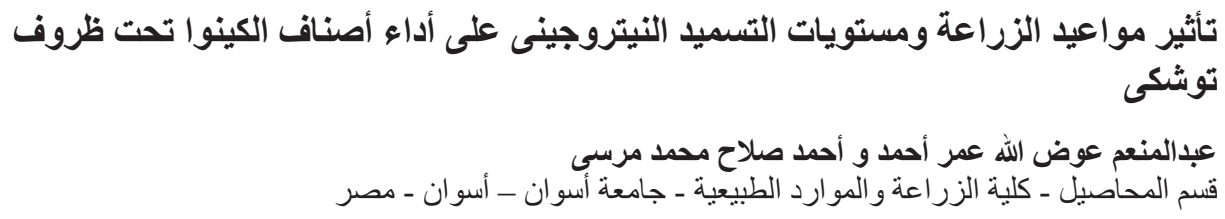

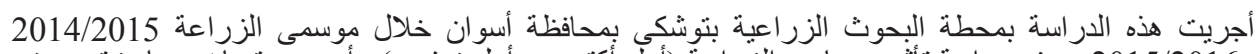

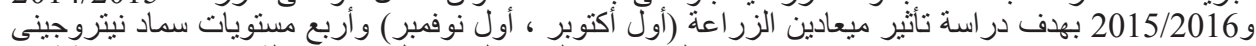

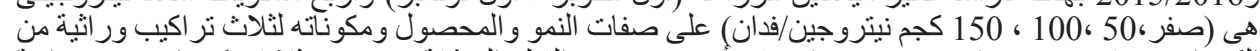

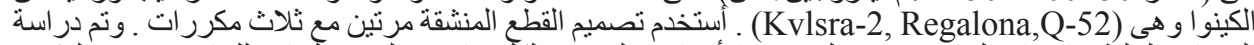

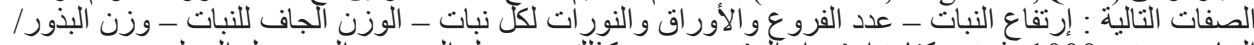

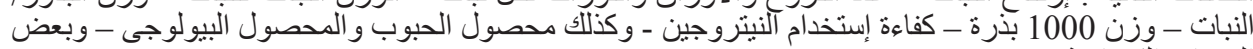
الصفات الكيماوية.

أوضحت النتائج وجود نأثثير معنوى للزر اعة فى أول نوفمبر على جميع صفات الار اسة مقارنة بالزر اعة فى أول

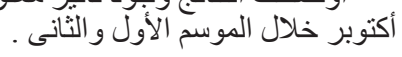

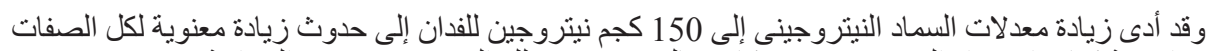

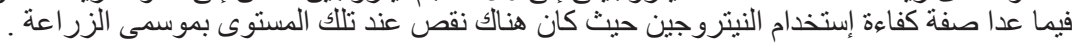

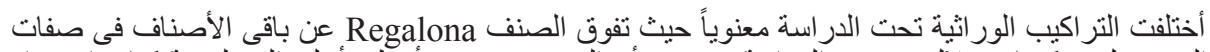

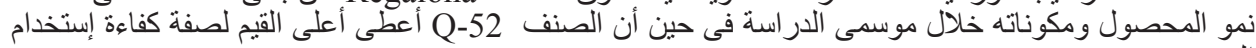
النيتزوجين . النوبر

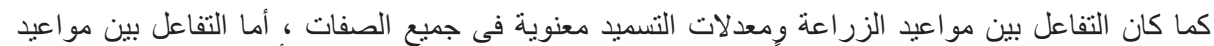

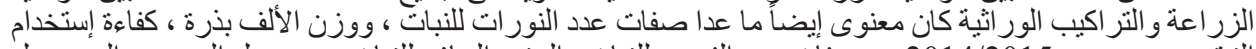

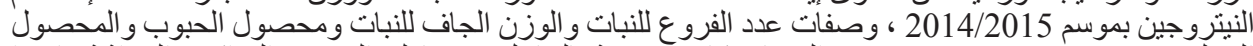

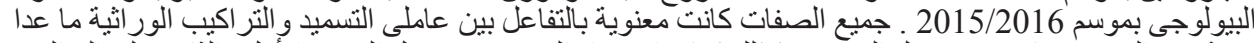

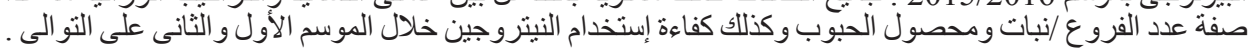

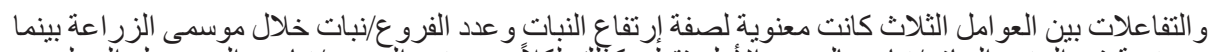

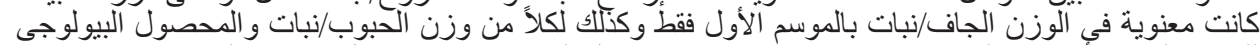

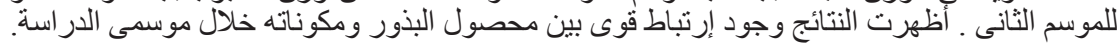

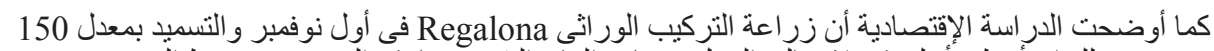

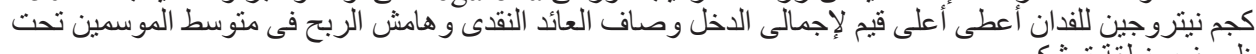

